\title{
Animal-inspired sensing for autonomously climbing or avoiding obstacles
}

\author{
William A. Lewinger ${ }^{\mathrm{a} *}$, Cynthia M. Harley ${ }^{\mathrm{b}}$, Michael S. Watson ${ }^{\mathrm{c}}$, Michael S. Branicky ${ }^{\mathrm{a}}$, Roy E. Ritzmann ${ }^{\mathrm{b}}$ \\ and Roger D. Quinn ${ }^{\mathrm{c}}$ \\ ${ }^{a}$ Department of Electrical Engineering and Computer Science, Case Western Reserve University, Cleveland, OH, USA; ${ }^{b}$ Department of \\ Biology, Case Western Reserve University, Cleveland, OH, USA, ${ }^{\circ}$ Department of Mechanical and Aerospace Engineering, \\ Case Western Reserve University, Cleveland, OH, USA
}

(Received 29 August 2008; final version received 9 December 2008)

\begin{abstract}
The way that natural systems navigate their environments with agility, intelligence and efficiency is an inspiration to engineers. Biological attributes such as modes of locomotion, sensory modalities, behaviours and physical appearance have been used as design goals. While methods of locomotion allow robots to move through their environment, the addition of sensing, perception and decision making are necessary to perform this task with autonomy. This paper contrasts how the addition of two separate sensing modalities - tactile antennae and non-contact sensing - and a low-computation, capable microcontroller allow a biologically abstracted mobile robot to make insect-inspired decisions when encountering a shelflike obstacle, navigating a cluttered environment without collision and seeking vision-based goals while avoiding obstacles.
\end{abstract}

Keywords: Whegs ${ }^{\mathrm{TM}}$; biologically inspired; autonomous navigation; mobile robotics; cockroach; antennae; microcontroller

\section{Introduction}

Animals use exteroceptive sensory information (tactile, vision, auditory, etc.) to perceive their environments and make intelligent decisions so that they can negotiate complex situations. This information is essential for them to move about their environment with a sense of knowledgeable intent to identify hazards or to decide which action to take next, which route to take when moving or the location of items of interest.

When faced with an option to climb over or tunnel under an object such as a shelf, a cockroach (Blaberus discoidalis) uses antennal contact with the obstacle as an aid in determining the course of action (Harley et al. 2008; Lewinger et al. 2005a, 2005b). The cockroach will almost always decide to follow the route discovered by both antennae if they agree. That is if both antennae touch an upper surface, the cockroach will climb; if both contact the underside, the insect will tunnel. Further decision making is necessary when the antennae are on opposite sides of the shelf, in which case experiments have shown that the insect will tunnel $43 \%$ of the time when in dark environments and $73 \%$ of the time when in light environments (Harley et al. 2008).

Bats use another sensory modality, ultrasonic echolocation, for detecting obstacles in their environment and locating prey when navigating and hunting (Horiuchi and Hynna 2001; Jones 2005). This non-contact, non-visual form of sensing allows the animal to better navigate and feed in low-light situations. Similar to cockroach antennae, information provided by the echolocation system, when integrated with other sensory data, processed by the animal's brain and coupled with its locomotion systems, allows it to exist in its world.

Obstacle-avoidance and obstacle-climbing behaviours are good models to provide a level of autonomy to mobile robots travelling in an obstacle-rich environment. Robots with the necessary mechanical mobility and sensory perception-decision making systems that enable these behaviours promise to navigate their environment with little or no human-operator interaction.

While there are many robotic platforms available for use as a base for implementing these sensing and reaction behaviours, Case Western Reserve University's Whegs ${ }^{\mathrm{TM}}$ II robot (Figure 1) from the Center for Biologically Inspired Robotics is a convenient choice. Its energetic and elegant Whegs ${ }^{\mathrm{TM}}$ locomotion method provides passive leg coordination and permits it to surmount large obstacles (as compared to its leg length) with little software control. Because its mobility gives it the option of climbing or circumventing obstacles, it is a good choice for this research.

Whegs ${ }^{\mathrm{TM}}$ II's climbing capability is superior to other robotic platforms such as RHex (Saranli et al. 2001) and Whegs ${ }^{\mathrm{TM}} \mathrm{I}$ because of the inclusion of a body-flexion joint

*Corresponding author. Email: william.lewinger@case.edu 


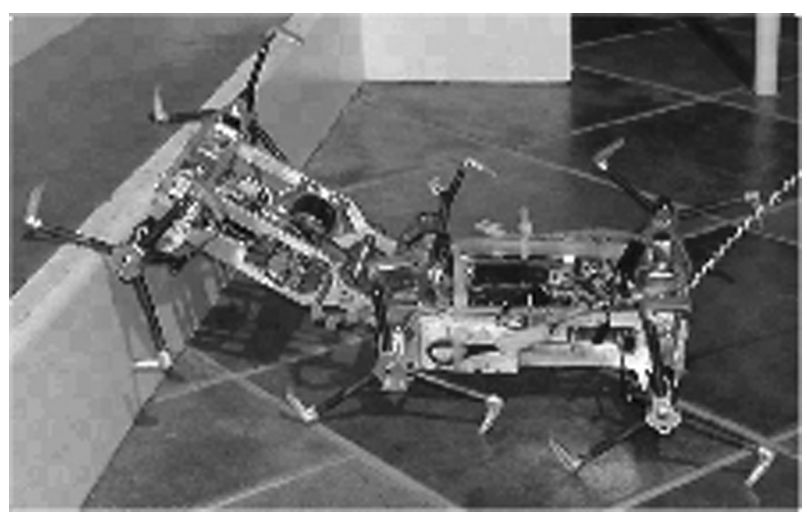

Figure 1. Whegs ${ }^{\mathrm{TM}}$ II uses three-spoke simplified legs that can reach the top of a barrier that is higher than the length of a spoke. Body flexion is used to rear the front half of its body.

which allows it to climb obstacles twice its leg length while avoiding high-centring situations. The natural climbing ability of the robot allows it to passively adjust its gait to run over obstacles higher than its leg length, while actively controlled body flexion adjusts its posture to allow it to surmount larger obstacles. The first behaviour described in this paper is with the addition of tactile antennae to detect shelflike obstacles, where climbing and tunnelling options are available. The second half of the paper reports the robot running over smaller obstacles and avoiding obstacles too tall to climb, using ultrasonic sensors in a bat-inspired configuration.

\section{Animal antennal use}

Cockroach antennae are flexible, multi-segmented structures. The most proximal segments are the scape and pedicel, both of which are jointed, controlled by a number of muscles and covered by a number of hairplates (Baba and Comer 2008; Okada and Toh 2000; Staudacher et al. 2005). When the antenna moves, hairs on these plates are deflected resulting in sensory signals, which encode information about the position of the antenna (Okada and Toh 2000, 2001). The most distal portion of the antenna, the flagellum, has additional hairs which are thought to sense the location of obstacle contact along its length (Camhi and Johnson 1999; Comer et al. 2003). It is likely that when contact occurs, combining the position of the antenna and the location of contact along the flagellum provides enough information for the cockroach to extrapolate the location of the obstacle.

However, antennae are able to sense more about an object than its location. Cockroaches are able to distinguish predators from conspecifics (Comer et al. 2003) and make decisions based on antennal information (Harley et al. 2008). When an insect encounters an object, the antennae touch first (Harley et al. 2008). In walking stick insects, this antennal contact is so important that gap-crossing behaviour only occurs once the substrate across the gap is discovered (Blaesing and Cruse 2004). For obstacles of moderate height, this contact results in changes in body attitude such that the insect is able to make a climbing trajectory (Figure 2; Pelletier and McLoed 1994; Watson et al. 2002). The success of this climbing trajectory depends on the insect's distance from the obstacle, which is sensed by the antennae (Harley et al. 2008).

In addition to sensing basic parameters of the obstacle such as its height and how far it is, the cockroach can also sense whether or not alternate routes exist. For instance, cockroaches are able to decide whether to climb over or tunnel under a barrier based upon their antennal contact information (Harley et al. 2008). If both antennae contact the top of the obstacle, the cockroach will climb over; if both contact the underside, the cockroach will tunnel under. If one antenna is on one side of the obstacle and one is on the other, the cockroach will move both to the same side and proceed to that side. The cockroach makes these choices based on its antennal contact pattern. In the dark when the antennae contact opposite sides of the shelf, the decision to climb or tunnel is relatively even (deciding to tunnel $43 \%$ of the time) because it is an exploratory/foraging behaviour. In the light, however, the insect tunnels to hide and chooses to tunnel $73 \%$ of the time (Jeanson and Deneubourg 2007; Okada and Toh 1998).

In an open environment the antennae make large sweeping motions (Bell 1991). These motions become smaller and move towards high-contrast objects in the visual field (Ye et al. 2003) or objects which are contacted by the antennae. Before they contact an obstacle, the antennae make large sweeping motions. While this would seem to be an inefficient strategy, there are several adaptations which increase efficiency. For instance, although the antennae have a large range of motion (Staudacher et al. 2005), only a fraction of that area is used for sensing. It is possible that the search area is restricted to a range in which the sensors are most effective. The antennal position at $60^{\circ}$ above the body axis results in the deflection of almost all of the sensory hairs on the dorsal scapal hairplate; beyond this point little information about the position of the antenna can be conveyed (Okada and Toh 2001). In the other direction, the angle below the body axis is limited by the ground. In addition, there is evidence that antennal movements 'focus' within an area in which they are likely to contact something relevant to the insect (Krause and Dürr 2004; Staudacher et al. 2005). To facilitate this likelihood, in stick insects, antennal oscillations seem to be coupled with walking speed (Dürr et al. 2001). The protraction phases of the antennae are coupled with the stance phase of the corresponding limb; however, this coupling is not exact. The antenna reaches the posterior extreme $0.2-0.25$ cycles prior to the corresponding leg (Dürr et al. 2001). These antennal movements, which occur out of phase, give the animal more information about its 


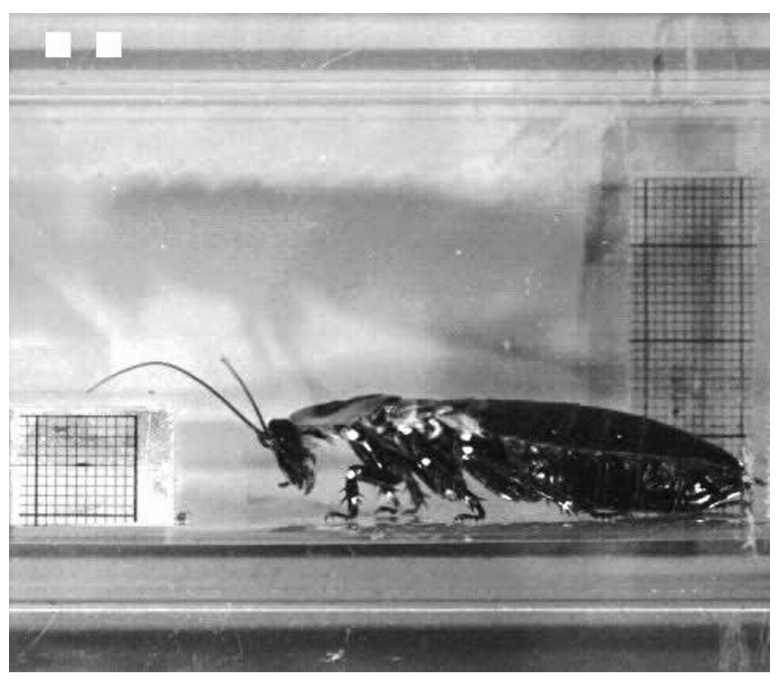

(a)

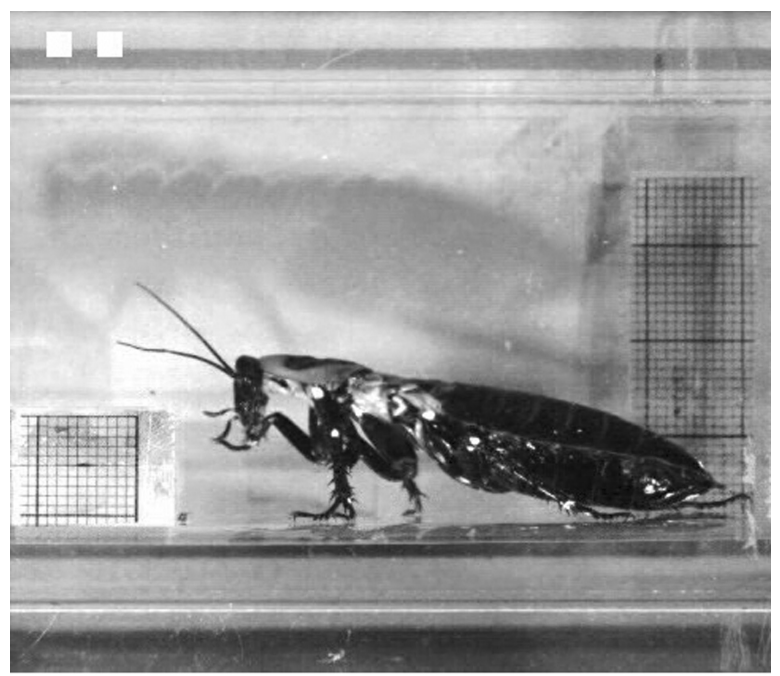

(b)

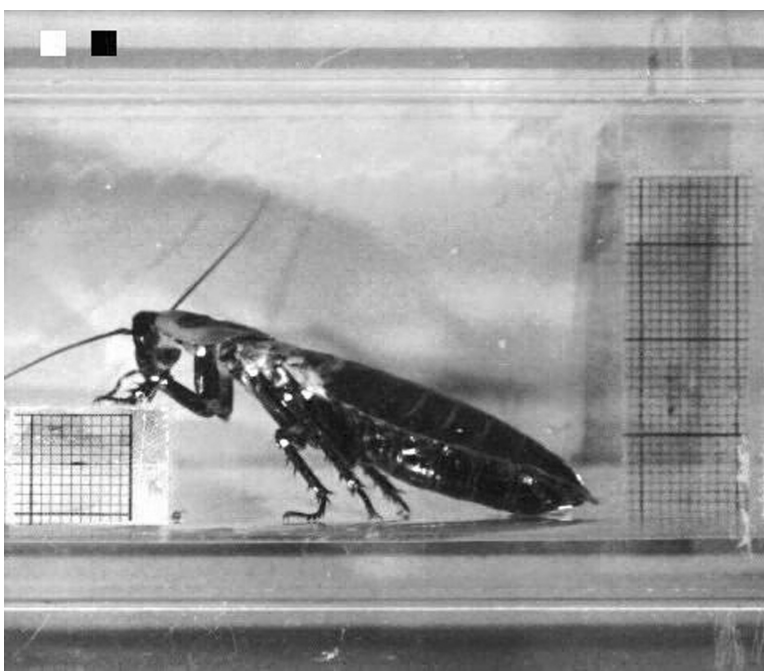

(c)

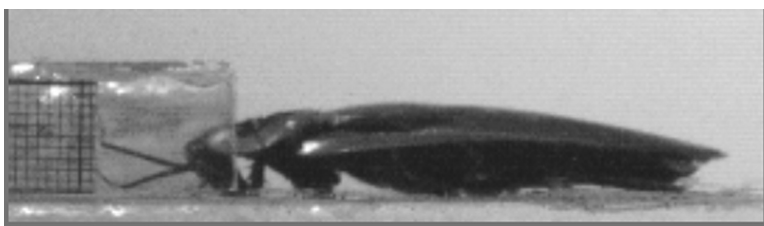

(d)

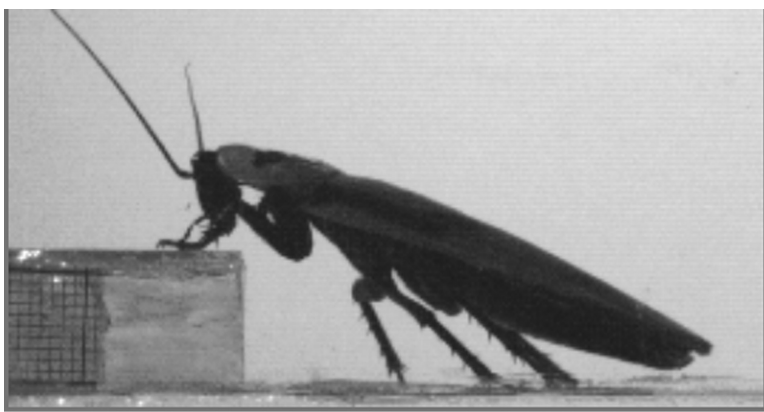

(e)

Figure 2. Pictures of the following behaviours: (a) approaching the block, (b) block-climbing swing, (c) block climbing, (d) shelf tunnelling and (e) shelf climbing.

environment than it would get from stationary antennae or if the antennae were in phase (Dürr et al. 2003).

\section{Whegs $^{\text {TM }}$ II}

Whegs ${ }^{\mathrm{TM}}$ II is a biologically inspired, six-legged mobile robot. It uses a single 90-W Maxon DC motor to propel all six of its legs. The drive motor is coupled with an integral 26:1, three-stage planetary transmission. The single-propulsion-motor design reduces the robot's weight and improves its power to weight ratio (Allen et al. 2003; Quinn et al. 2003).

A major advantage of legs over wheels is their ability to gain discontinuous footholds; i.e. they alternate between the stance phase, in which they contact the substrate, and the swing phase, in which they do not. This aspect is beneficial on irregular, discontinuous terrain. The Whegs ${ }^{\mathrm{TM}}$ vehicle's three-spoke wheel-leg appendages abstract the principles of a cockroach's tripod gait (Watson and Ritzmann 1998) while rotating at constant speed.

The tripod gait is not always suitable for a hexapod. In fact, when climbing larger barriers, cockroaches often move their leg pairs in phase (Watson et al. 2002). Whegs ${ }^{\mathrm{TM}}$ II incorporates compliant mechanisms in all six of its axles, which accomplish phase changing passively (Allen et al. 2003; Quinn et al. 2003). These mechanisms cause them to run in a nominal tripod gait (Watson and Ritzmann 1998) but passively adapt their gaits to irregular terrain (Figure 1). 


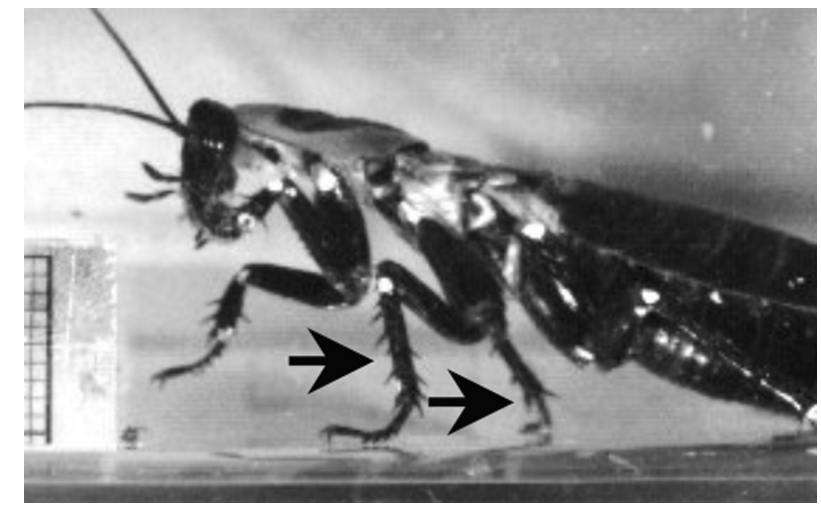

Figure 3. Cockroach rears its body prior to climbing.

This compliance captures much of what the cockroach accomplishes through actions of its distal leg joints. Hence, the vehicle will have more feet in contact with the ground and will be more stable. These passive leg adjustments are similar to the preflexes described by Loeb et al. (1999).

A cockroach enhances its climbing abilities by changing its body posture before and during the climb over an obstacle (Watson et al. 2002). For example, it performs a rearing movement prior to climbing obstacles that are taller than it could normally reach with its front legs (Figure 3). A Whegs ${ }^{\mathrm{TM}}$ vehicle cannot rear up using its middle legs. However, it can accomplish the goal of raising the front legs higher by rotating a body joint upward. Cockroaches have a thoracic body-flexion joint, located between their front and middle thoracic segments, that enables them to bend the front half of their bodies downward. This body flexion enables it to extend its front legs downward and grasp the substrate in a favourable configuration for pulling itself up and over the obstacle.

Whegs ${ }^{\mathrm{TM}}$ II has a body-flexion joint that is collocated with its middle axle and is actuated by a radio-controlled (R/C) servo. The front of its body can be flexed up (Figure 1) or down $30^{\circ}$ from the neutral position. In Figure 1 it is rearing up the front half of its body so that its front legs can reach the top of a step while the rear and middle legs drive it forward. Using its body-flexion joint Whegs ${ }^{\mathrm{TM}}$ II can climb obstacles that are higher than twice its leg length. As shown in Figure 1, this configuration permits the leg to get a foothold on an obstacle that is higher than the length of a spoke. Whegs ${ }^{\mathrm{TM}}$ II uses $10-\mathrm{cm}$ long spokes that move in the sagittal plane (Figure 1). Each leg is constructed with rubber-coated spring steel, which offers abundant traction and radial compliance.

There are robots with spoke legs which preceded Whegs $^{\mathrm{TM}}$. The PROLERO hexapod robot used a singlerotating-spoke design for each of its six legs. It used one motor for each leg, to rotate its legs in a circular motion to enable walking and turning (Martin-Alvarez et al. 1996). RHex expanded upon this single-spoke concept by incor- porating compliant legs and a more complex control architecture for insect gaits, climbing and body movements (Saranli et al. 2001). Whegs ${ }^{\mathrm{TM}} \mathrm{II}$, in contrast, is steered by two small $\mathrm{R} / \mathrm{C}$ servos that are electrically coupled to rotate the front and rear legs in opposite directions. This in combination with the body and geometry of Whegs ${ }^{\mathrm{TM}}$ II gives it a turning radius of 1.25 body lengths and is similar to the manner in which cockroaches turn, except that all three pairs of their legs are engaged in turning their bodies (Jindrich and Full 1999; Mu and Ritzmann 2005).

\section{Implementation of mechanical antennae to navigate shelflike obstacles}

Antennae are primary sensors for much of the orientation behaviour exhibited by cockroaches and other insects when they move on the ground. This tactile orientation behaviour can be used for wall following (Camhi and Johnson 1999), identification of a predator (Comer et al. 2003) and object-guided orientation (Okada and Toh 2000). Antennae may also be used for navigation in complex environments.

Robots inspired by insects and other animals have previously been designed with physical antennae and tactile sensors to navigate their environment, as in the work by Brooks (1989), Cowan et al. (2005), Hartmann (2001) and Lee et al. (2008); the last three works employed the use of a single tactile element rather than a pair. These other works describe investigations into obstacle and wall detection by searching in the horizontal plane, whereas the work presented in this paper uses antennae searching in the sagittal plane to detect shelflike obstacles. The video associated with this research won an ICRA 2005 award (Lewinger et al. 2005b).

This section describes the implementation of mechanical antennae for an agile, mobile robot to navigate an environment containing shelflike obstacles that can be climbed over or tunnelled under.

\section{Design of mechanical antennae}

A mechanical set of antennae were designed for attachment to the Whegs ${ }^{\mathrm{TM}}$ II robot (Figure 4). The antennae are two fibreglass rods $457-\mathrm{mm}$ long with a diameter of $3 \mathrm{~mm}$ attached to the output horns of multi-point injection (MPI) 350-HP R/C servo motors and covered with Interlink Model 408 force-sensing strips on the top and bottom. The servo motors are attached to hinged brackets that allow the antennae to rotate backward. In the event the robot elected to tunnel under the shelf while one antenna was still above it, this feature would allow the antenna and servo assembly to rotate backward rather than become damaged or impede the robot's progress. The hinged sections are held in place by magnets to prevent this backward-rotation movement during normal operation. 


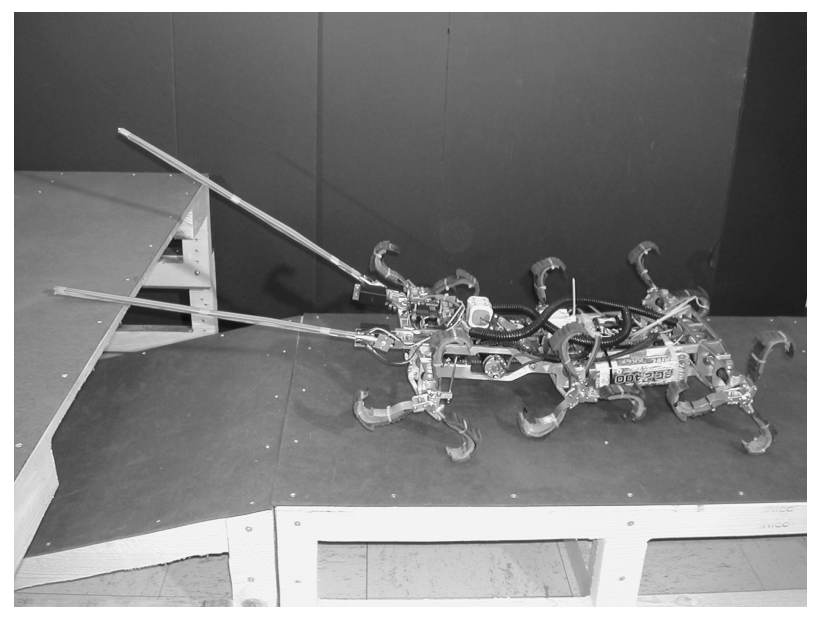

Figure 4. Whegs ${ }^{\mathrm{TM}}$ II with attached mechanical antennae.

\section{Climbing/tunnelling system model}

The goal of this implementation is to use a pair of headmounted antennae such that the robot will autonomously decide whether to climb or tunnel when presented with a shelflike obstacle. A state-based system model was developed to capture the desired behaviour of the robot and antennae. Initially, the robot begins in a 'seek' state. In this state the robot moves forward, and the antennae oscillate at different rates from one another and change direction when the upper or lower limit is reached. The oscillation rates for each antenna are maintained at constant speeds with varying direction and do not have sinusoidal velocity.

Antennal contact is determined by measuring signal deviation in a voltage divider network using force-variable resistive sensors. The force sensors are connected to a 4.8$\mathrm{V}$ dc microcontroller-based analogue to digital (A/D) converter via $10-\mathrm{k} \Omega$ pull-up resistors.

When an antenna registers contact with the target, forward motion of the robot is stopped; the antenna making contact stops oscillating (while the other antenna continues), and a timer is started. This gives the second antenna time to make contact by preventing the robot from driving past a point at which contact can occur. The timer is used so that the robot does not wait indefinitely for two antennae to detect an obstacle. Situations could arise due to mechanical damage or failure (i.e. a missing or an immobile antenna) or when only one antenna is able to sense the obstacle due to orientation with the target. As the second antenna contacts the shelf or as the timer expires, a decision to climb or tunnel is made.

The decision is always made in favour of a path determined by two antennae on the same side of the shelf, so that both antennae sensing the top of the shelf will cause a climbing behaviour (scenario 1), and both antennae contacting the underside of the shelf will cause the robot to tunnel (scenario 2). If one antenna senses the target and if the timer expires before the other antenna makes contact, the robot will move towards the side that the first antenna contacted (scenario 4). The other scenario (scenario 3) occurs when one antenna contacts the top of the shelf and the other antenna contacts the underside. In this situation, the robot will exhibit a tunnelling behaviour. A tunnelling behaviour was chosen for this scenario, as it was observed that cockroaches opt to tunnel $90 \%$ of the times in well-lit environments when faced with similar situations (Harley et al. 2008).

\section{Simulation}

The system model was simulated in MATLAB to examine the relationship between system and environmental parameters. The following parameters were varied to test for interdependence: antennal oscillation rates, antennal sweep ranges, robot speed, initial distance to target and second antenna contact timeout value. Each parameter had five values selected for simulation, while the other parameters were fixed at the middle values for each test. Table 1 shows the selected values.

The 'contact timeout' parameter represents a distance and, consequently, a time after the first antenna contacts the obstacle and within which the second antenna must also contact the target. If the second antenna does not contact the target within that distance (time period), a single-antenna contact decision is made. For all simulations, antenna length was $457 \mathrm{~mm}$; head and ramp heights were equal at $102 \mathrm{~mm}$; and the time interval between samples was $0.05 \mathrm{~s}$.

While it was observed in insect experiments that cockroaches oscillated their antenna at speed-dependent rates, yet different from one another, the exact frequencies and phase differences in antennal oscillations were not quantified due to extreme variability. Additionally, in stick insects, antennal oscillations seem to be coupled with walking speed (Dürr et al. 2001). The protraction phases of the antennae are coupled with the stance phase of the corresponding limb; however, this coupling is not exact. The antenna reaches the posterior extreme $0.2-0.25$ cycles prior

Table 1. Two-antennae simulation parameters.

\begin{tabular}{lccccc}
\hline Parameter/trial & 1 & 2 & 3 & 4 & 5 \\
\hline Right antenna rate (deg/s) & 2.0 & 4.0 & 6.0 & 8.0 & 10.0 \\
Left antenna rate (\% of R) & 80 & 85 & 90 & 95 & 100 \\
Antennae $y_{\min }$ (in./cm) & 1.0 & 1.5 & 2.0 & 2.5 & 3.0 \\
& 2.5 & 3.8 & 5.1 & 6.4 & 7.6 \\
Antennae $y_{\max }$ (in./cm) & 5.0 & 5.5 & 6.0 & 6.5 & 7.0 \\
& 12.7 & 14.0 & 15.2 & 16.5 & 17.8 \\
Robot speed (in./s) & 3.0 & 6.0 & 9.0 & 12.0 & 15.0 \\
Target distance (in./cm) & 24 & 30 & 36 & 42 & 48 \\
& 61 & 76 & 91 & 107 & 122 \\
Contact timeout (in./cm) & 2.0 & 4.0 & 6.0 & 8.0 & 10.0 \\
& 5.1 & 10.2 & 15.2 & 20.3 & 25.4 \\
\hline
\end{tabular}


to the corresponding leg (Dürr et al. 2001). These antennal movements, which occur out of phase, give the animal more information about its environment than it would get from stationary antennae or if the antennae were in phase (Dürr et al. 2003). As such, the dissimilar oscillation rates between the two antennae were implemented in the robot simulations.

In order to maintain a balance between high-resolution sensing and low power consumption, an acceptable robot speed to antennal oscillation rate needed to be implemented.
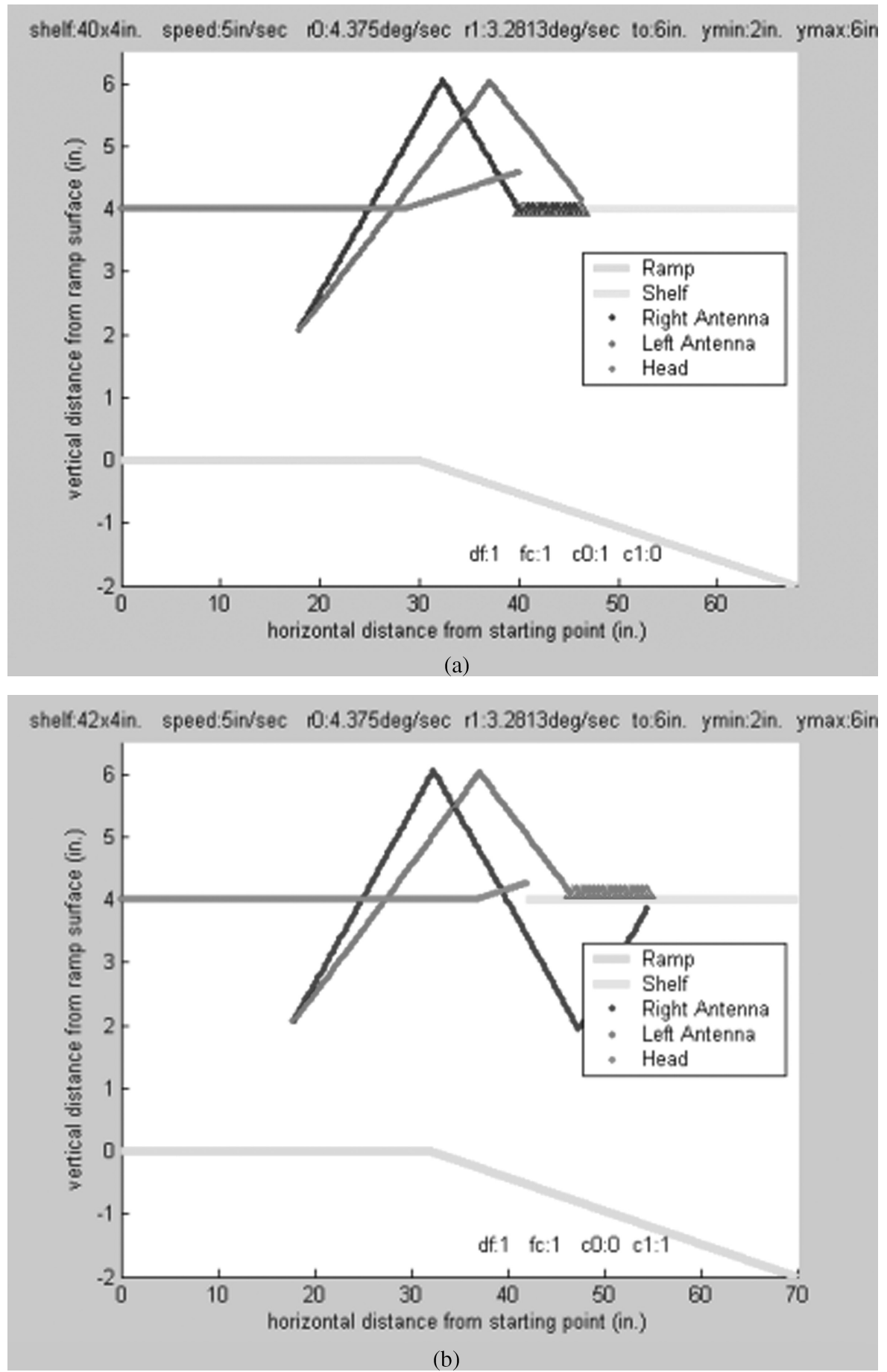

Figure 5. Right oscillation rate (blue line $)=0.875 \times$ (robot speed); left oscillation rate (red line) $=0.75 \times($ right oscillation rate $) 1$ robot head (fuchsia), shelf (cyan); approach path and ramp (green line). Initial distance from shelf: (a) $1.02 \mathrm{~m}$, (b) $1.07 \mathrm{~m}$, (c) $1.12 \mathrm{~m}$, (d) 1.17 $\mathrm{m}$, (e) $1.22 \mathrm{~m}$, (f) $1.27 \mathrm{~m}$ and (g) $7.62 \mathrm{~m}$. Note: Vertical and horizontal axes represent height and distance, respectively. Numeric caption denotes starting distance from the shelf. This figure is available in color online. (Continued) 

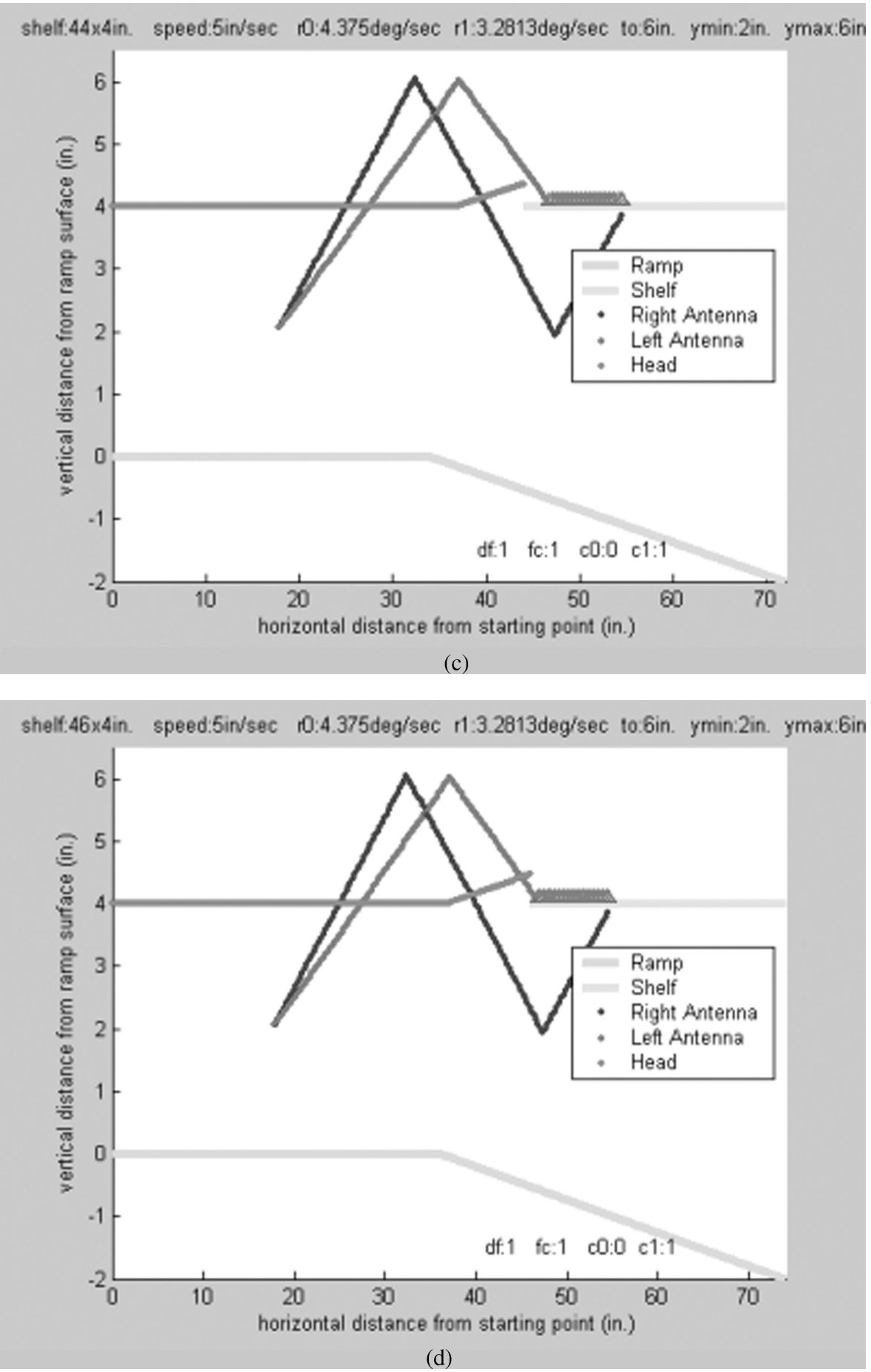

Figure 5. Continued

In an ideal case this value should be infinitely small, indicating an extremely high antennal oscillation rate. However, this was both impossible and impractical.

Through simulation trials, a good balance between low oscillation rate and high coverage area was having the right antenna oscillate at $87.5 \%$ of robot speed and the left an- tenna oscillate at $75 \%$ of the right antenna rate. These values provided a compromise between motor exertion and coverage of the vertical plane (Figure $5 \mathrm{~g}, 7.62 \mathrm{~m}$ image) and were implemented using a BrainStem microcontroller. Figure 5 shows the results for varying starting distances from the shelf. The dark blue line is the path of the right antenna; 

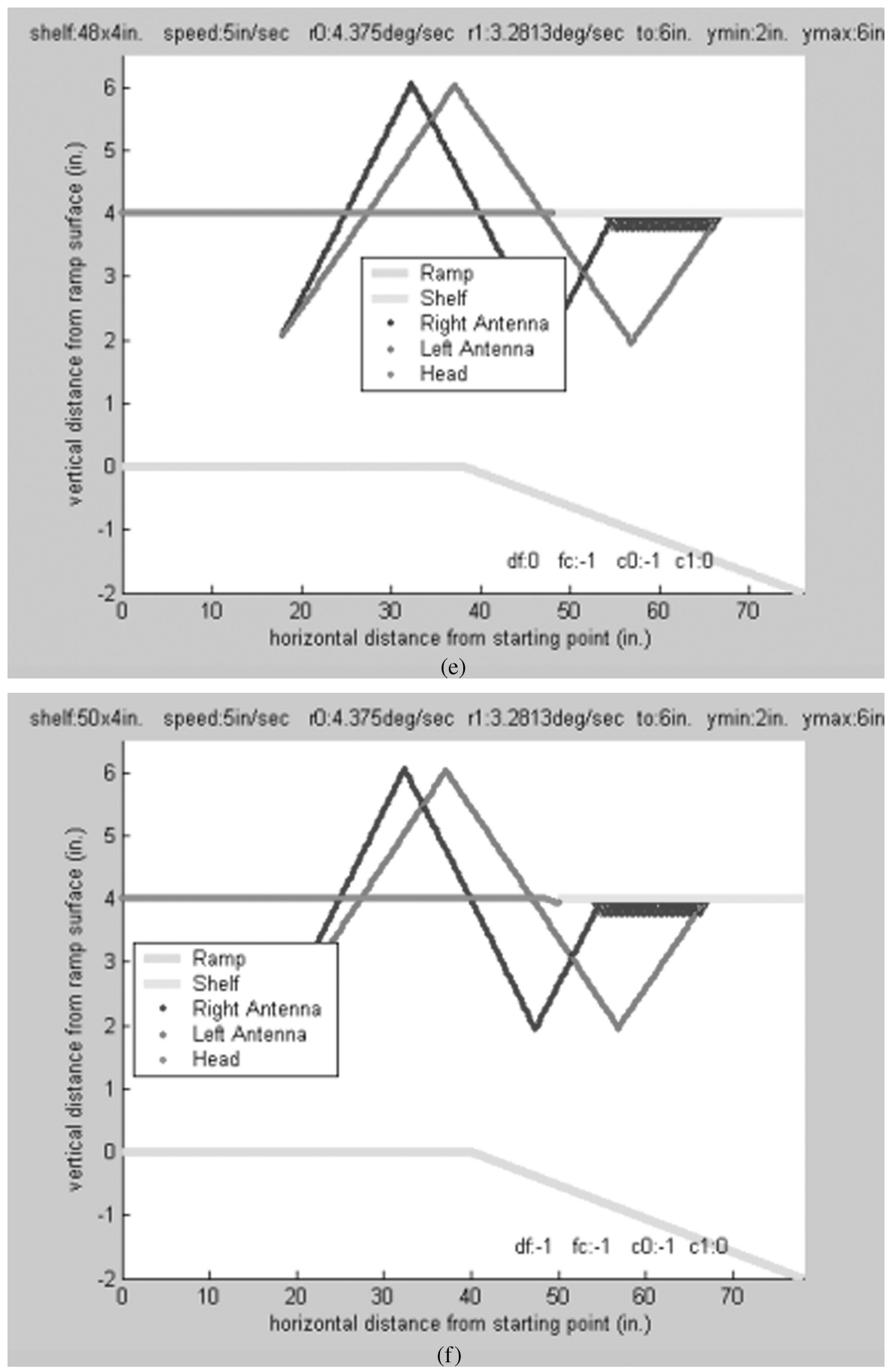

Figure 5. Continued

the red line is the path of the left antenna. Heavy blue and red symbols indicate that the corresponding antenna had made contact with the shelf and had stopped oscillating. The fuchsia line is the path of the robot 'head'. It remains at a height of $102 \mathrm{~mm}$ until a decision to climb or tunnel is made, when it deflects up or down in the image. The shelf and the approach ramp positions are shown in cyan and green, respectively.
One observation from the simulations is that antennal position becomes a function of distance when related to robot speed. As the physical distance is limited to a maximum of $1.32 \mathrm{~m}$ in the test environment, a minimum oscillation rate was implemented, preventing the antennae from oscillating at the rate of zero. This changes the system to become a function of time rather than distance. By waiting different times before initiating forward motion 


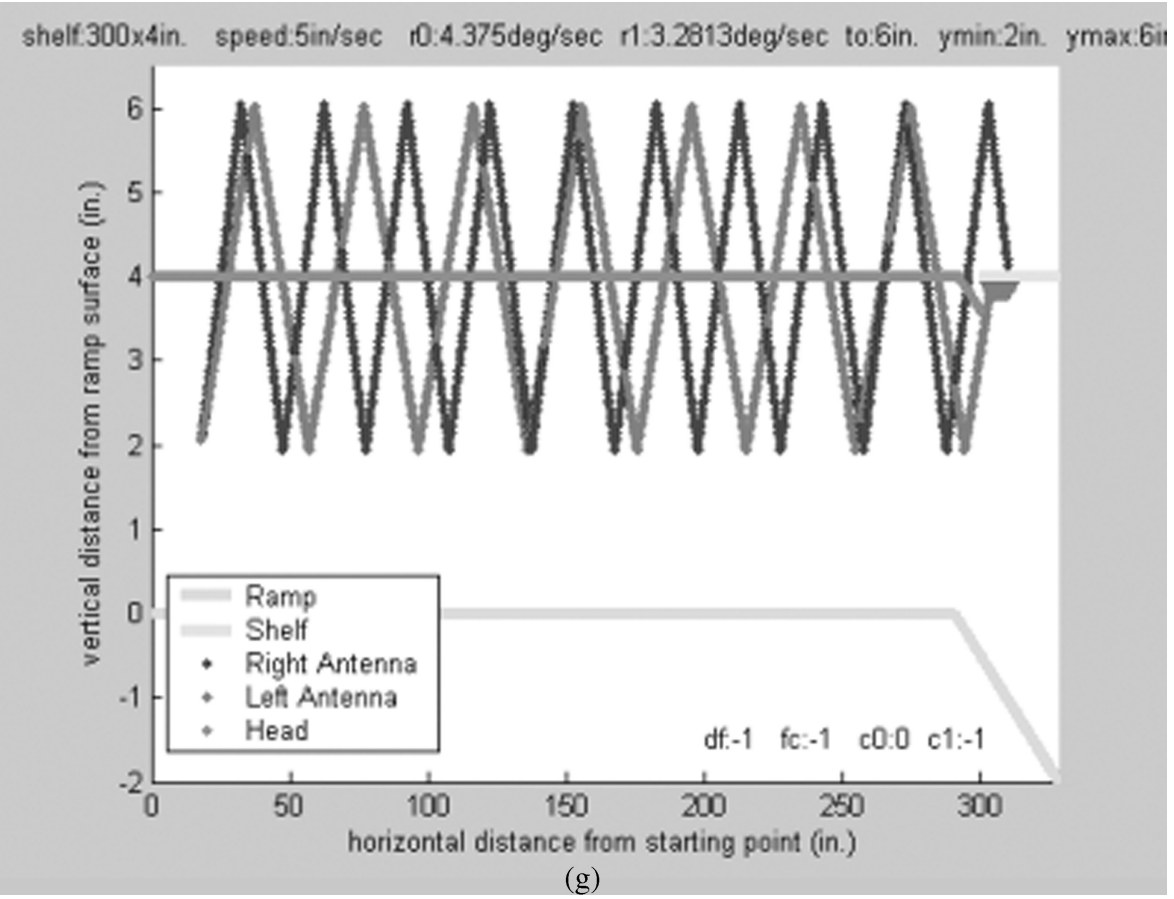

Figure 5. Continued

towards the obstacle, different antennal contact scenarios can be explored.

\section{Test environment}

The physical environment for the Whegs ${ }^{\mathrm{TM}}$ II robot antenna experiments (Figure 6) is a $0.61 \mathrm{~m}$ wide, $1.83 \mathrm{~m}$ long, raised approach path with a $95 \mathrm{~cm}$ ramp descending at $15.5^{\circ}$. In addition, a $1.22 \mathrm{~m}$ wide, $0.61 \mathrm{~m}$ long shelf that straddles the ramp provides a climbing option as well as a tunnelling option for travel. The shelf has an adjustable height but was set to correspond with the height of the robot's 'head'.

\section{Software behaviour implementation}

The Whegs ${ }^{\mathrm{TM}}$ II robot is typically radio-controlled with a three-DOF wireless FM transmitter $(72 \mathrm{MHz}$, Channel

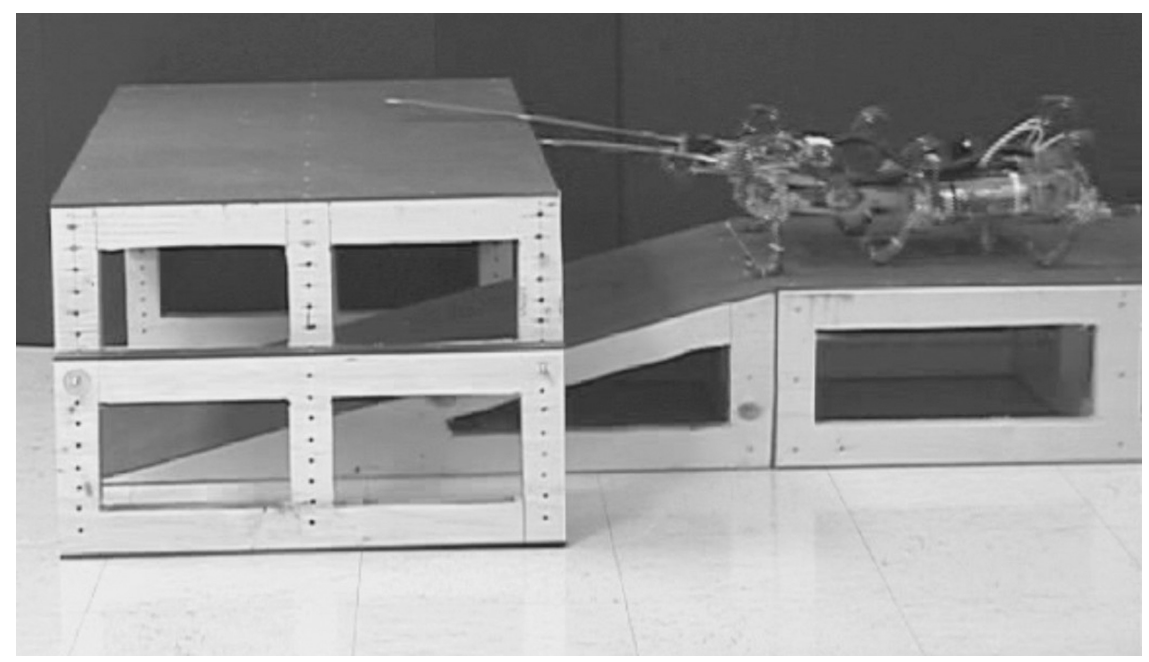

Figure 6. Whegs ${ }^{\mathrm{TM}}$ II with initial antennae design during stand-based testing. A new, more robust design was then implemented that allowed Whegs ${ }^{\mathrm{TM}}$ II to navigate the test ramp without causing false-positive readings (Figure 6). This allowed full testing of the behaviour. With the unpredictability of how the antennae would contact the shelf, it was difficult to capture each of the four scenarios. 


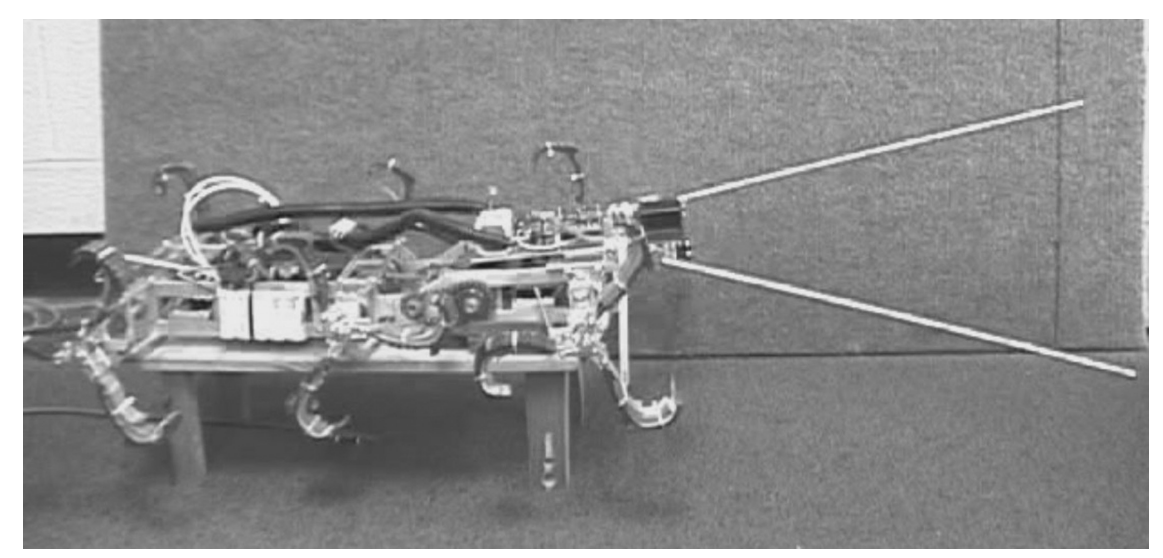

Figure 7. Whegs ${ }^{\mathrm{TM}}$ II on the test ramp with antennae contacting the shelf on the top and bottom (scenario 3 ).

50). The transmitter allows an operator to control speed (forward and reverse), steering (left and right) and flexion of the body joint (up and down). For these experiments, $\mathrm{R} / \mathrm{C}$ commands were intercepted en route to their respective motors and interpreted by an Acroname BrainStem GP 1.0 microcontroller (a low-computation capable microcontroller), which re-created the speed and steering signals but impeded the body-joint flexion signal from the user. A second BrainStem GP 1.0 module was used to actuate the antenna motors and sense antennal contact via an onboard $\mathrm{A} / \mathrm{D}$ converter. The two microcontrollers were connected to one another via an $\mathrm{I}^{2} \mathrm{C}$ communications bus.

The implemented behaviour began by reading the robot drive speed signal from the $\mathrm{R} / \mathrm{C}$ receiver. This signal was used to determine antennal oscillation rates with the boundary of $8-23 \%$ of the maximum possible robot drive speed. While in the bounded region, actual drive speed was used for antenna oscillation rate calculations; outside the region, the floor or ceiling value was used. Each antenna continued oscillating until object contact was sensed, at which time the robot drive motor signal was impeded to stop further forward movement. A timeout period of $1 /(\%$ robot speed) given in milliseconds was initiated when contact was sensed, providing a period inversely proportional to speed, during which the other antenna continued to oscillate. When the second antenna made contact with the shelf, or the timeout period expired, oscillation of the second antenna was stopped, and the decision to climb/tunnel was made. The robot speed signal was retransmitted, and the body-flexion joint was actuated in the direction of the decision for a time of $\tau=(\%$ robot speed $) \times 128 / 100 \mathrm{~s}$. The body-flexion joint was then straightened for $\tau$ s and then actuated in the opposite direction for $\tau$ s. Finally, the body-flexion joint was again straightened. This series of movements would allow the Whegs ${ }^{\mathrm{TM}}$ II robot to properly tunnel under or surmount a shelf.
Table 2. Whegs ${ }^{\mathrm{TM}}$ II ramp trial results.

\begin{tabular}{lc}
\hline Result & Number of times \\
\hline Scenario 1 & 1 \\
Scenario 2 & 1 \\
Scenario 3 & 5 \\
Scenario 4 & 2 \\
False positive contact & 2 \\
Missed shelf & 1 \\
\hline
\end{tabular}

The programmed behaviour faithfully follows the system model described in the climbing/tunnelling system model section.

\section{Results}

The original antennal design measured the difference in the commanded and actual positions to determine contact with the shelf. However, due to repeated sensing of false positive readings because of antennal inertia while the robot was running, additional testing was performed with the robot on a stand. The stand allowed leg movement and body flexion while removing the influence of body motions on the antennae during running (Figure 7).

A series of tests was performed by manually bending the antennae to simulate contacting an obstacle. This was performed to test each of the four scenarios: scenario 1 in which both antennae were on top; scenario 2 in which both antennae were underneath; scenario 3 in which the antennae split by the shelf; and scenario 4 in which there was single-antenna contact. Split antennae and single-antenna contact scenarios were performed for each permutation of left and right antennae contact configurations. The testing was video-taped and showed that the programme modules were performing as expected, and the climb/tunnel decision was correctly being made and executed. Each 


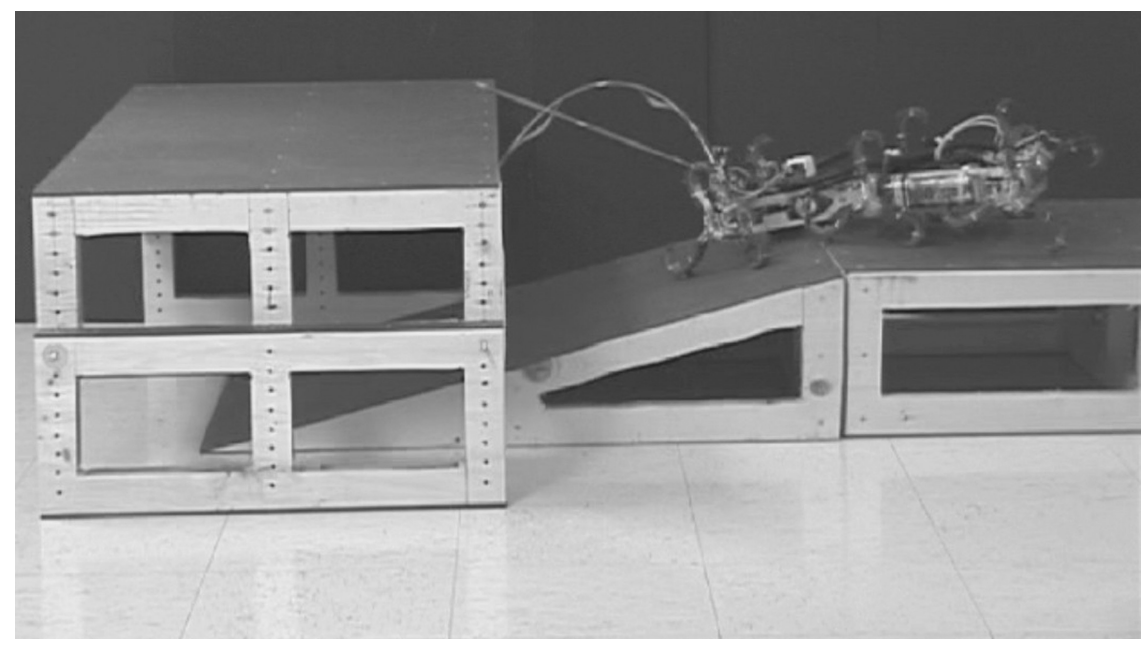

Figure 8. A unique situation in which one of the antennae speared the shelf.

scenario followed the sequence of programmed states and actuated the body-flexion joint correctly to achieve the desired climb/tunnel response. Tests were then performed with the updated antennal design (given in the section 'Design of mechanical antennae') with the robot moving in the test arena described in the section 'Test environment'.

Since the antennae are constantly moving - and at varying rates - there is a limited potential for both antennae to contact the same side of the object. This results in more cases of scenario 3 (split antennae) but increases the likelihood of contacting the shelf (Figure $5 \mathrm{~g}, 7.62-\mathrm{m}$ image).

During two of the trials, false positive contacts were observed. For these trials, an antenna speared the shelf and caused severe flexing of the antenna, which signalled a contact situation (Figure 8). The trials were stopped before viewing any resulting behaviour action, and the contact force thresholds for the antennae were increased after the second incident. In one trial, the robot walked under the shelf without making contact with it. After this trial, the range of antennae oscillations was increased to cover more area above the shelf.

For each trial in which the shelf was detected, the robot successfully navigated the shelf by performing the appropriate body-joint flexion behaviour and autonomously moving beyond the obstacle by tunnelling or climbing.

\section{Implementation of non-contact sensing to navigate cluttered environments}

In addition to tactile antennae described above, cockroaches can also use their visual system and will direct their antennae towards objects within the visual field (Ye et al. 2003). The same holds true for a mobile robot. Non-contact sensing can avoid antennae accidents and impede motion such as shown in Figure 8. In this section we explore ultra- sonic sensors independent of tactile antennae for obstacle avoidance.

Insects such as crickets (Horchler et al. 2003; Lund et al. 1998) and small animals like the little brown bat (Horiuchi 2005; Horiuchi and Hynna 2001; Shi and Horiuchi 2004) have binaural hearing that relies on intensity (level) and phase differences in the sounds received by each hearing sensor. Intensity measurements are used, since the separation of the hearing organs is very small. Larger creatures like owls (Carr and Konishi 1990) and people (Martin 1995) measure the time delay between the signals received in each ear to determine azimuth. The temporal differences of sound stimuli from each ear are compared to locate the azimuth of the source.

This implementation of non-contact sensing is relatively long-ranged and is different from some previous robotic implementations, such as the Electrolux Trilobite and iRobot Roomba autonomous vacuum cleaners. The Electrolux Trilobite uses ultrasonic sensing to detect nearcollision situations in which the cleaner is within $2.54 \mathrm{~cm}$ of an obstacle, while both units have variations that use downward-pointing infrared sensors to detect the presence of a drop-off. In both cases the sensors are used as shortrange, reactionary devices rather than long-ranged, pathplanning tools.

\section{Design of the ultrasonic sensor pod}

Inspired by the interaural time difference (ITD) method of sound location, as used by larger animals, and using a selfgenerated sound pulse, as in the echolocation of bats (Horiuchi 2005; Horiuchi and Hynna 2001; Shi and Horiuchi 2004), a binaural sensor pod was created using ultrasonic sensors. 


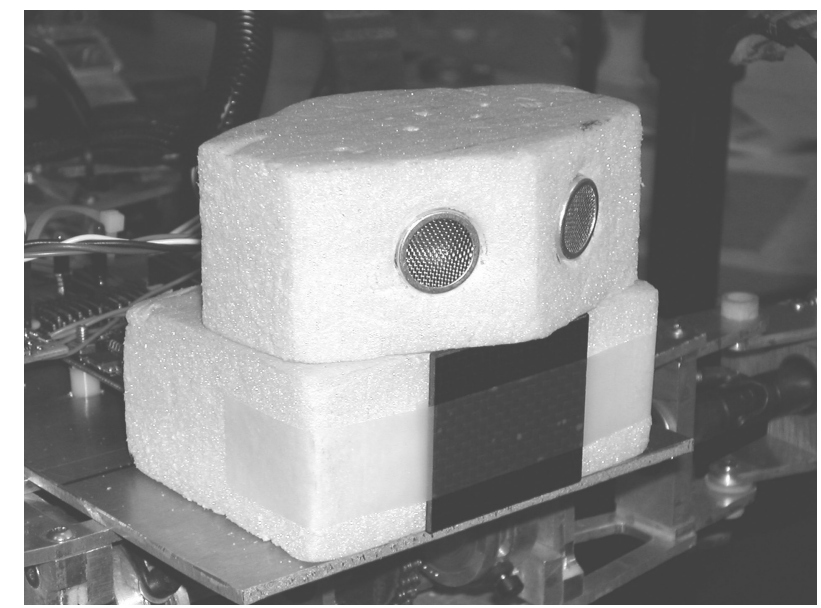

Figure 9. Binaural ultrasonic sensor pod with single ultrasonic emitter (located behind the black square) and angled dual receivers above.

Devantech SRF08 ultrasonic range-finding units (Devantech Ltd, Norfolk, UK) were used as the basis for the sensor pod and have a single emitter and a single receiver mounted to a small board containing signal-processing electronics. They were chosen for their balance of accuracy, range, speed and cost. The sensors were then modified to separate the emitter and receiver units from the electronics board so that they could be repositioned. The design of the sensor pod (Figure 9) uses one of the emitters, located centrally, and both of the receivers. This is unique to most implementations of ultrasonic sensors, with the exception of the work done by Horiuchi (Horiuchi 2005; Horiuchi and Hynna 2001; Shi and Horiuchi 2004). The receiver units are each angled outward from the sagittal plane by $22.5^{\circ}$.

Using a single, central emitter eliminated the possibility of conflicting echo signals, which could have occurred if two emitters were used. Having two receivers separated by a distance allows detected objects to be located in a planar environment by determining the distance between the object and each sensor (Feiten et al. 1994; Lawitsky et al. 1995). By angling the wide-field receivers outward two overlapping receptive fields were created (Bank 2002a, 2002b). This allowed fewer receivers to detect the same object and locate its position rather than using many more narrow-field receivers.

The binaural ultrasonic sensor pod was mounted to the front of the Whegs ${ }^{\mathrm{TM}}$ II mobile robot (Figure 10). Using this robot for the sensor platform allowed larger objects to be sensed and avoided and smaller objects to be climbed over, something that wheeled robots of the same size cannot do. Additionally, the position of the ultrasonic sensor pod allowed for small objects that could be easily surmounted to be ignored and consequently climbed over. This is unique to other implementations of ultrasonic sensing, such as the 'batmobile' (Horiuchi 2005), that are only

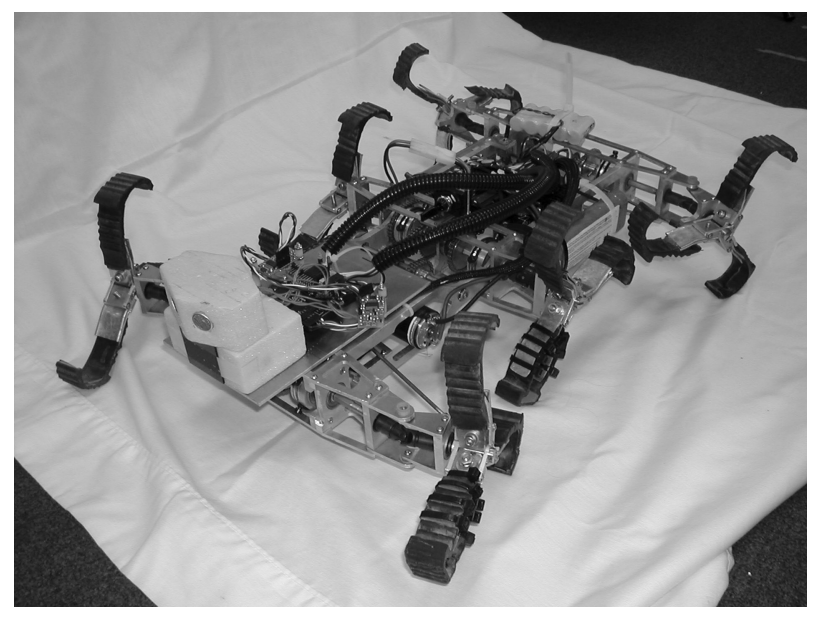

Figure 10. Whegs ${ }^{\mathrm{TM}}$ II mobile robot with binaural ultrasonic sensor pod mounted on the front.

capable of avoiding all obstacles and do not distinguish between large (to be avoided) and small (can be climbed over) obstacles.

Two obstacle detection and avoidance behaviours were created to locate objects and avoid them. The first behaviour allowed semi-autonomous operation of the robot and used a simple method of detecting objects within one of three zones: left, centre and right. Objects sensed in the left or right zone caused the robot to steer away in the opposite direction. Centrally located obstacles stopped the robot's forward progress and required an operator to reverse direction and choose a new heading.

The second behaviour provided the robot with full autonomy. It used a 2-m sensor range and determined the azimuth of obstacles in the overlapping, central sensing region, using the ITDs of the two receivers. Once the location of an object was calculated, steering and drive speed were adjusted proportionately based on the position and distance of the object.

\section{Ultrasonic sensor characteristics}

While there is available information on the SRF08, experiments were conducted to determine the actual range capabilities and the sensor envelope. These measurements were required to fully characterise the sensors before integrating two of them into the sensor pod.

The first set of experiments was performed to determine the sensor envelope of a single, unmodified SRF08 (with both the emitter and receiver still attached to the electronics board). Two sets of planar tests were performed to calculate a three-dimensional representation of the envelope. Each of the planar tests had the Devantech SRF08 firmly mounted $1.2 \mathrm{~m}$ from the floor. The first test had the sensor mounted so that the emitter and receiver were positioned side by side. The second test had the sensing elements positioned 
one above the other. These tests were designed to determine the $x y$-plane envelope and the $y z$-plane envelope.

To find the sensor envelope, a 120 -cm long tube 15 $\mathrm{cm}$ in diameter was placed at various locations in front of the SRF08. At each location the Devantech sensor was queried whether it could detect the tube or not. Initially, the tube was moved at $30-\mathrm{cm}$ increments to grossly determine the envelope. Then, smaller movement increments were used to get a more accurate image of the sensing ranges and angles. Points at which the SRF08 was able to detect the tube were plotted to define the boundary of the sensor envelope (Figure 11).

The Devantech SRF08 detected the tube at distances up to $7.6 \mathrm{~m}$. The initial sensing field was an arc of approximately $90^{\circ}$ for a distance of about $3 \mathrm{~m}$. Beyond $3 \mathrm{~m}$ the envelope maintained a fixed width of about $6 \mathrm{~m}$. Tests performed with the sensor mounted vertically showed similar results, indicating a conical sensor envelope. Other experiments were performed with tubes of smaller diameters down to $5 \mathrm{~cm}$. The SRF08 was able to detect the smaller object throughout the entire envelope.

After the envelope was determined for the single, unmodified Devantech SRF08 ultrasonic sensor, the sensors were altered for use as a binaural set. The emitter and receiver were unsoldered from the electronics board and reconnected to the board by $15-\mathrm{cm}$ long cables. This

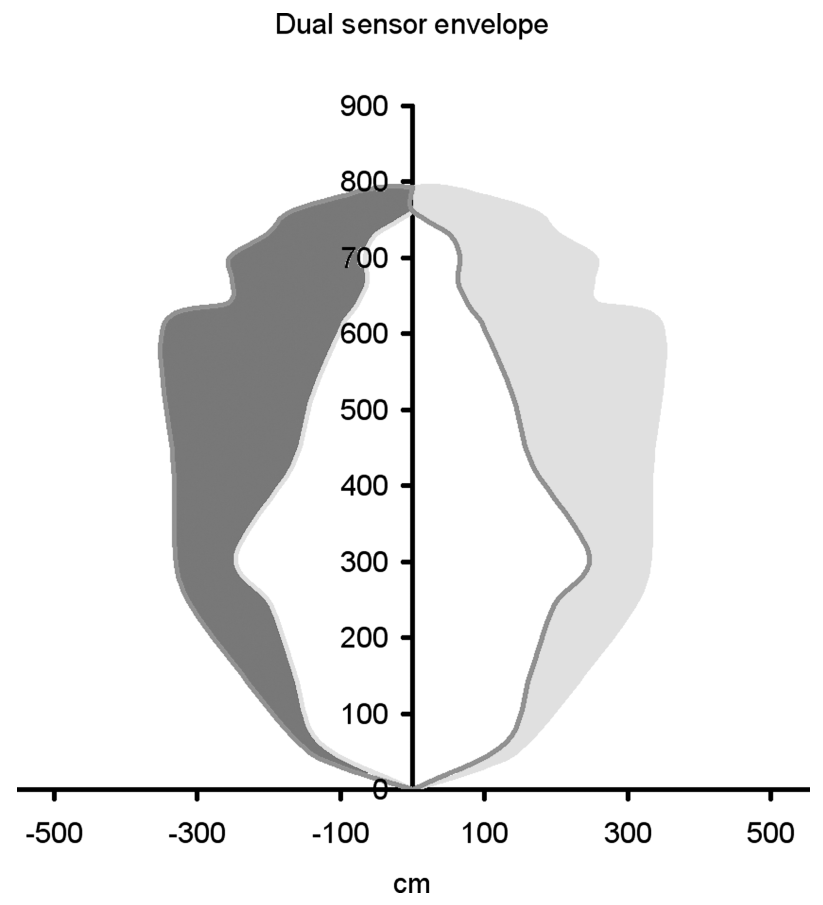

Figure 11. Sensor envelope for the binaural ultrasonic sensor pod with a single emitter with angled dual receivers. The red area is able to be sensed by the left receiver only, and the green area is able to be sensed by the right receiver only. The middle white area can be sensed by both sensors due to the overlapping sensing fields. This figure available in colour online. allowed the emitter and receiver to be mounted at new positions and angles, relative to one another. The emitter and receiver from a single SRF08 were used as the central emitter and right-side receiver. A second SRF08 was modified so that the receiver formed the left-side receiver, and the emitter was removed, which did not affect the operation of the sensor.

Once modified, the emitter and two receivers were mounted in foam (Figure 9). The receivers were separated by $35 \mathrm{~mm}$ and angled $22.5^{\circ}$ from the sagittal plane. The emitter was centrally located, $50 \mathrm{~mm}$ below the receivers.

Additional experiments were then performed to determine the new $x y$-plane sensor envelope for the binaural ultrasonic sensor pod. The new experiments were conducted in a similar manner with a $120-\mathrm{cm}$ long tube $15 \mathrm{~cm}$ in diameter at various positions in front of the sensor pod. The left and right sensor envelopes mirrored one another and expanded the detection angle near the pod from $90^{\circ}$ to about $105^{\circ}$ (Figure 11) for a distance of about $3 \mathrm{~m}$. Beyond $3 \mathrm{~m}$ the combined envelope detected objects in a $7-\mathrm{m}$ wide path out to a range of about $8 \mathrm{~m}$.

While the receivers are capable of detecting echoes from angles of $\pm 45^{\circ}$, the emitter cannot send ultrasonic pulses over the range of $135^{\circ}$ (the full possible sensory range of two receivers angled $45^{\circ}$ apart). As a result, the sensor envelope for the binaural sensor pod is limited to $105^{\circ}$.

By using two receivers at a splayed angle, three sensory zones were created. There are two outer zones in which only a single sensor can detect an object (coloured areas at the bottom of Figure 11) and a central zone in which both sensors receive echoes from the same target (white area at the bottom of Figure 11).

Using a single emitter with a pair of receivers widens the sensor envelope, improves detection performance and reduces sensing time. Activating two emitter/receiver pairs at the same time could cause spurious echoes that would confuse the sensors. To eliminate this possibility, each sensor pair would need to emit and receive echoes before activating the other sensor pair. This would double the time required for both sensors to detect objects. A single emitter with two receivers eliminates the echo interference and long detection time issues.

\section{Implementation and behaviour}

The binaural ultrasonic sensor pod (described above) was mounted to the front of the Whegs ${ }^{\mathrm{TM}}$ II chassis on which it had an unobstructed view of the area ahead of the robot (Figure 10). This mounting placed the emitter approximately $15 \mathrm{~cm}$ from the ground with the receivers $20 \mathrm{~cm}$ from the ground.

Initially, ground reflections were observed approximately $15 \mathrm{~cm}$ in front of the robot. Attempts to remedy the situation included additional shielding between the ultrasonic emitter and receivers in the form of a horizontal 
plate of carbon fiber. This, however, was insufficient. In the end, a carbon fibre filter was placed over the emitter (the black square in Figure 9) to attenuate the broadcasted signal. This reduced the intensity of the emitter signal and allowed the sensors to ignore almost all ground reflections.

An Acroname BrainStem microcontroller (Acroname, Boulder, CO, USA) was mounted behind the sensor pod and was used to trigger the ultrasonic emitter and read echo signals from each of the receivers.

The ultrasonic range-finders continuously reported the distance of the first echo every $100 \mathrm{~ms}$. This time was chosen to be larger than the maximum 65 -ms timeout value representing the farthest distance the range-finders could detect objects $(11 \mathrm{~m})$.

Two separate behaviours were programmed into the robot. The first was used in a semi-autonomous mode, where an operator drove the robot and where the binaural sensor pod detected obstacles and corrected the robot's course and/or speed, overriding the operator's commands. The second was fully autonomous operation, where the robot determined its own steering angle and drive speed based on the location and distance of detected obstacles without any input from an operator.

For semi-autonomous operation the BrainStem also interpreted radio control commands from the $\mathrm{R} / \mathrm{C}$ receiver as initiated by the operator. The three $\mathrm{R} / \mathrm{C}$ channels that control speed, steering and the body-flexion joint were all interpreted by the BrainStem and then modified, as needed, based on the detection of sensed obstacles. For these experiments, however, the body-flexion joint was not used, as the trials were performed on a flat surface.

The $\mathrm{R} / \mathrm{C}$ channel signals were interpreted by reading the pulse width $(0.9-2.1 \mathrm{~ms})$ from the $\mathrm{R} / \mathrm{C}$ receiver with the level transition detecting capabilities of the BrainStem digital input/output (I/O) ports. The pulse width was converted to an 8 -bit value with $0.9 \mathrm{~ms}$ corresponding to 0 and $2.1 \mathrm{~ms}$ corresponding to 255 . This represented the full range of $\mathrm{R} / \mathrm{C}$ servo values that could be transmitted to the Whegs ${ }^{\mathrm{TM}}$ II steering and body-joint motors and the drive motor speed controller to actuate and move the robot. When no obstacles were detected in the path of the robot, the operator commands were passed on to the robot motors without modification.

During semi-autonomous mode (Function 1) an obstacle warning distance of $75 \mathrm{~cm}$ was set as a software threshold for when the avoidance behaviour would take action. If an obstacle appeared in the left field within $75 \mathrm{~cm}$, the robot would turn to the right to avoid a collision. This was accomplished by replacing the operator's intended steering control value and creating a full-right turn signal to be sent to the steering servo motors instead. The operator's intended signal was still interpreted by the BrainStem but then ignored in favour of the behaviour's control signal. Once the object was no longer detected within range, the operator's signal was once again sent to the steering servo motors. Similarly, obstacles appearing in the right field would cause the robot to steer to the left until the obstacle was no longer detected.

Function 1

threshold $=75 \mathrm{~cm}$

if(left sensor $<$ threshold and right sensor $>$ threshold)

turn full right

if(left sensor $>$ threshold and right sensor $<$ threshold)

turn full left

if(left sensor $<$ threshold and right sensor $<$ threshold)

stop forward motion

This avoidance behaviour created the ability for the robot to follow a wall. If the operator drove the robot forward while steering it towards a wall, the robot would approach the wall until a potential collision was detected. Then, the obstacle-avoidance behaviour would steer the robot away from the wall while it still moved forward. When the wall was no longer in range, the operator's steering command would resume, and the robot would once again head towards the wall.

If both left and right ultrasonic receivers detected an obstacle, indicating the presence of something in the centrefield, forward motion was stopped. In this case the operator could fully steer the robot between the left and right extremes but could only drive in reverse.

While the threshold distance was set to $75 \mathrm{~cm}$, the actual distance at which actions were taken varied with the angle the obstacle face had with the sagittal plane. The more acute the angle, the closer the robot came to the obstacle before an autonomous action was taken. In two cases, the angle of the wall with the sagittal plane was very small (less than $15^{\circ}$ ), and glancing impacts occurred.

For the second, fully autonomous behaviour (Function 2) object distance was measured as the time (in microseconds) for the ultrasonic signal to reach an object and return. Microsecond measurements were used to provide finer resolution than $\mathrm{cm}$ measurements $(\sim 29 \mu \mathrm{s} / \mathrm{cm})$. A sensory threshold of $2 \mathrm{~m}$ was set, and objects beyond $2 \mathrm{~m}$ were ignored.

When an obstacle was detected three calculations were made: average range to the target for both receivers (rangeAvg), the closest sensed distance by which obstacleavoidance intensity was determined (rangeDist) and a difference in distance values sensed by the two receivers (rangeDiff).

Function 2

sensing threshold (threshold) $=2000 \mathrm{~mm}$ sensor separation (separation) $=35 \mathrm{~mm}$ $\operatorname{sgn}(\mathrm{val})=1$ if val $>0$ else -1 rangeAvg $=$ (right sensor + left sensor) $/ 2$ rangeDist $=$ threshold - rangeAvg rangeDiff $=$ (right sensor - left sensor) $/$ separation if (abs(left sensor - right sensor) < separation) steerVal $=128+($ rangeDist $/$ rangeDiff $) * \operatorname{sgn}($ rangeDiff $)$ 
else if (right sensor $<$ left sensor)

steerVal $=128+($ threshold - right sensor $) / 92$

rangeAvg = right sensor

else if (right sensor $<$ left sensor)

steerVal $=128+($ threshold - right sensor $) / 92$

rangeAvg $=$ left sensor

driveVal $=128+(\operatorname{rangeAvg} * 128 /$ threshold $)$

If a detected obstacle is sensed by both receivers and if the difference in the measured distances is less than the distance between the placement of the two sensors, both receivers are detecting the same object. In this case, steering is inversely proportional to the distance of the object and its location to the side of the robot's path. For example, objects that are far away or far to the side of the robot create a smaller steering angle from the current path.

If detected distances differ by more than the distance between the sensors; then more than one obstacle is detected, and the sensor with the closer object has priority. In this case steering is still inversely proportional to the distance to the obstacle, but the difference between the left and right sensors is not taken into consideration.

Speed is proportional to obstacle distance. The farther away an obstacle is located, the faster the robot moves forward. If an object is in front of the robot and cannot be avoided by steering, forward speed is reduced to zero, and the robot stops. If the obstacle is moved further away or removed entirely, the robot resumes its movement automatically. The current behaviour doesn't allow the robot to drive in reverse.

Once steering and speed values are calculated, the commands are autonomously sent to the steering servo motors and drive motor speed controller to move the robot.

\section{Experiment scenarios}

Experiments for the semi-autonomous behaviour were performed in a $1.5-\mathrm{m}$ wide, $8.5-\mathrm{m}$ long section of hallway. The floors were covered with $30-\mathrm{cm}$ square vinyl tiles. The walls were formed of painted concrete blocks with $10-\mathrm{cm}$ tall vinyl strips at the base.

Two series of experiments for this behaviour were conducted. The first was a set of wall-following tests. The second was a set of two unique obstacle courses. The obstacle course contained six $60-\mathrm{cm}$ tall cardboard cylinders $12 \mathrm{~cm}$ in diameter placed at random along the length of the course.

For the wall-following scenario, the operator drove the robot forward while steering towards either the left or the right wall. In the obstacle course scenario, the operator drove the robot generally forward, allowing autonomous course corrections to avoid collisions. However, some operator steering was used to intentionally choose paths towards obstacles. Additionally, operator driving and steering was used in the obstacle course scenario when an obstacle was detected in the centrefield of view. During these cases, the operator controlled the robot to drive in reverse until the obstacle was no longer in range and chose a new heading when resuming forward motion.

For the fully autonomous behaviour similar wallfollowing and obstacle course experiments were performed. These experiments took place in a square room (4.9-m long, $3.7-\mathrm{m}$ wide) with similar flooring and walls as the semiautonomous experiments.

Wall-following trials were conducted about the perimeter of the room. For later experiments, obstacle courses with three and four $60-\mathrm{cm}$ tall cardboard cylinders $12 \mathrm{~cm}$ in diameter were created. Additional experiments were also performed in both the wall-following and obstacle course scenarios with low $(5-\mathrm{cm}$ tall) obstacles for the robot to climb over.

Each fully autonomous trial began with placing the robot in the test environment. The robot was then manually activated, and no further operator actions were taken until the trial ended and the robot was deactivated. The robot decided its heading and speed through its environment based solely on sensor inputs and its programmed behaviours.

\section{Results}

Eight experiments were conducted with the semiautonomous behaviour. Six trials were performed for the wall-following scenario, and two trials were performed with the obstacle course scenario. For the second set of trials one run was performed in each of two unique obstacle courses.

One run is shown in Figure 12(a). The actual path taken by the robot is shown in blue. Short red lines indicate the times in which the operator directed the robot towards the wall, but the obstacle-avoidance behaviour steered it away from the impending collision. Six autonomous course corrections were made by the behaviour, and no wall collisions occurred. For all six trials, 31 of 33 collisions with the wall were avoided ( $94 \%$ success).

Figures 12(b) and 12(c) show semi-autonomous runs through obstacle courses (red circles). The obstacleavoidance behaviour avoided 25 out of 26 collisions $(96 \%$ success). In the first obstacle course the robot collided with the wall once and required operator action to correct the situation. The obstacle-avoidance behaviour performed the correct steering action; however, the robot was too close to the wall when it was detected, and it was too late to prevent the collision. There were no collisions with walls or other obstacles during the second obstacle course.

Forty-five experiments were conducted with the fully autonomous behaviour: 32 wall-following trials and 13 obstacle-avoidance trials.

During the wall-following trials the robot was placed near a wall and activated. If the robot was close enough to the wall it approached the wall and steered away such that the angle with the wall became increasingly shallow. Also, 


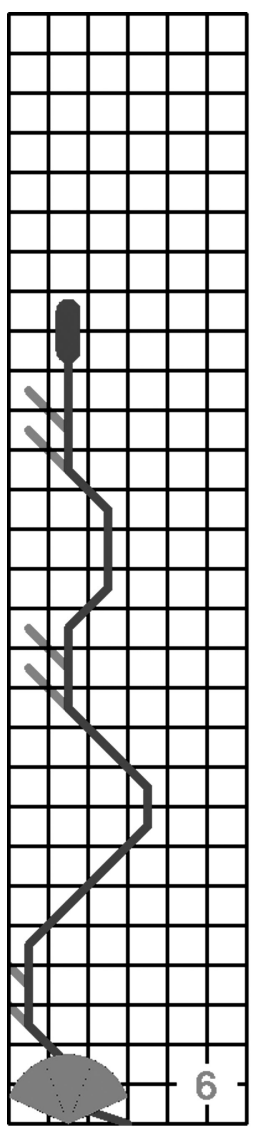

(a)

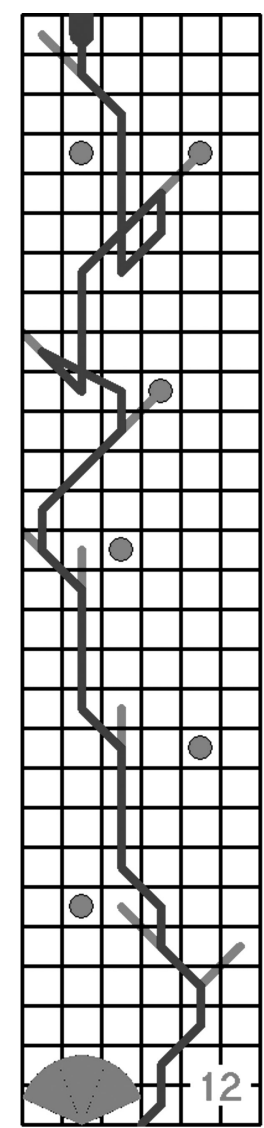

(b)

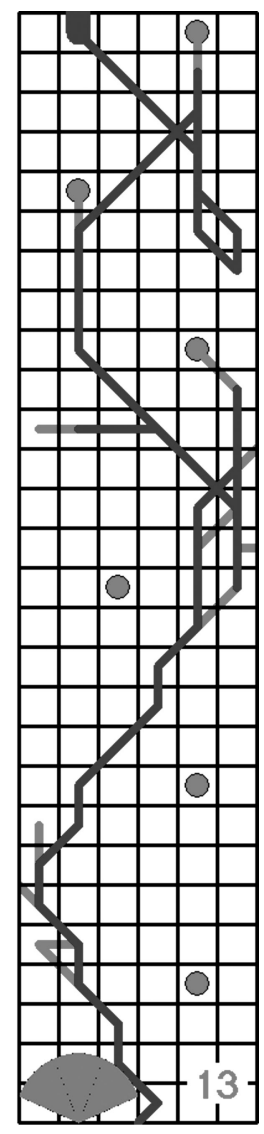

(c)
Figure 12. Robot paths for (a) wall following and (b, c) two unique obstacle courses. Short red branches show the commanded path that wasn't chosen. Numbers indicate times that an autonomous course correction was made to avoid collision. The robot moved from bottom to top in each case. This figure is available in colour online.

as the robot came closer to the wall the speed decreased. The robot continued to follow the wall until it found a corner, at which time it stopped, since there was no path it could take according to the programmed behaviour. If the robot started farther from a wall it could successfully turn away from an encountered corner and continue about the enclosed room following the perimeter. Throughout these trials no wall collisions occurred.

Five of the wall-following trials involved a low $(5-\mathrm{cm})$ obstacle that Whegs ${ }^{\mathrm{TM}}$ II was easily capable of walking over. During four of these trials, the ultrasonic sensor field detected the low obstacle and considered it impassable, at which time the robot came to a halt. After modifying the sensor field by blocking the lower portion of the sensor envelope, the robot successfully followed the wall while ignoring the presence of the low obstacle, which was easily surmounted. The robot did not collide with a wall during any of these trials.
The second series of trials involved six runs through a course with three tall, cylindrical obstacles, four runs through a course with four tall, cylindrical obstacles and three runs through the four-obstacle course with an additional low obstacle.

During these trials, as with the wall-following scenario, the robot steered away from obstacles proportional to their distances in front of the robot and away from the robot's path. In a few cases the robot approached an obstacle headon and was not able to steer away from it. In these cases the robot stopped in front of the obstacle without contacting it.

As with the low-obstacle scenario during the wallfollowing trials the robot initially considered the obstacle something to be avoided and steered away from it. Once the sensor field was modified to ignore low obstacles, the robot successfully climbed over the low obstacle in the other two trials.

It should be noted that during the fully autonomous wall-following trials and obstacle course trials, no collisions with either walls or obstacles occurred (100\% success).

\section{Simultaneous vision-based goal seeking and ultrasonic sensing for obstacle avoidance}

The previous sections discussed two different methods for detecting and climbing or avoiding different types of obstacles. While obstacle navigation is an important behaviour for mobile robots, it is insufficient for more advanced navigation tasks in which a desired destination is present. To improve on the non-contact obstacle-avoidance work, the ultrasonic sensor pod was updated, and a colour camera was added to Whegs ${ }^{\mathrm{TM}}$ II for vision-based goal seeking. The addition of multi-modal sensing is also motivated by our animal models. Biological systems rarely use a single form of sensing to perform a task. For example, cockroaches use vision and tactile antennae, and bats combine vision and ultrasonic echolocation to negotiate obstacles.

\section{Updated ultrasonic sensor pod design}

To correct minor issues experienced with the original ultrasonic sensor pod design, such as physical robustness, the inability to ignore low, climbable objects and better attenuation of spurious signals, a new design was implemented (Figure 13).

This new sensor pod used open-cell foam tubes to shroud the ultrasonic emitter and receiver units. These tubes acoustically attenuated the emission and reception of the ultrasonic pulses, which produced a shorter, narrower sensing field. Since much of the original sensor field was already being ignored, this modification improved the range of sensing to being confined only to areas of interest near the robot.

A new addition to the sensor pod was a CMUcam2 unit with an OV7620 Omnivision CMOS colour camera on 


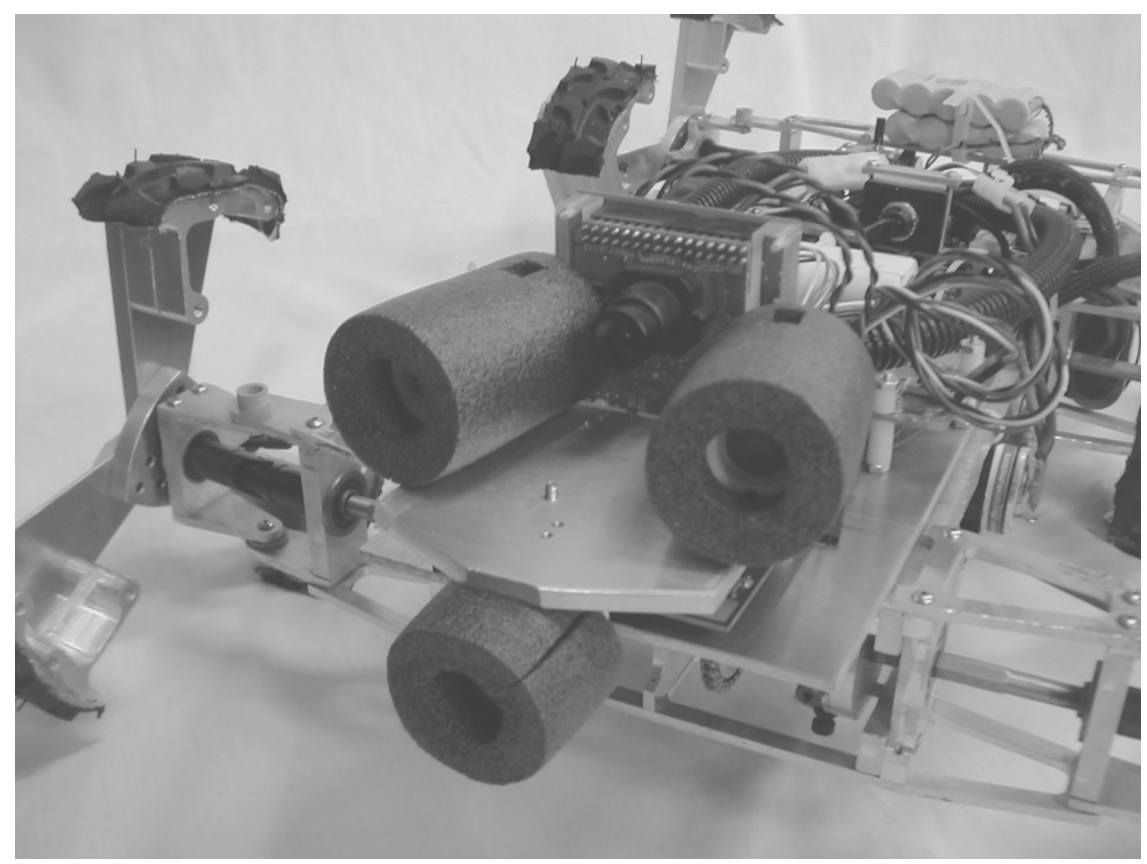

Figure 13. Whegs ${ }^{\mathrm{TM}}$ II robot with updated binaural ultrasonic sensor pod including a single ultrasonic emitter, angled dual receivers and a colour camera used for vision-based goal seeking and simultaneous obstacle avoidance.

a chip (Acroname, Inc., Boulder, CO, USA). The camera unit was able to detect colour-based objects and generate pan and tilt signals for R/C servo motors. Additionally, its RS-232 serial interface allowed connection to a microcontroller to provide full information on coloured object's two-dimensional position and number of occupied pixels in the field of view.

\section{Goal-seeking and obstacle-avoidance behaviours}

New behaviours were created to handle goal seeking and obstacle avoidance. All of these behaviours were implemented on a single BrainStem microcontroller, located within the new sensor pod.

The built-in demo mode of the CMUcam2 was used to generate pan and tilt signals to control R/C servo motors. In this mode, the camera is trained on a target colour on start-up. Pan and tilt signals are then generated to position the target colour in the centre of the field of view. For this implementation, however, only the pan signal was used. The signal was connected to a digital input of the BrainStem microcontroller, where its pulse width was measured in a manner identical to reading the operator command for the semi-autonomous mode described in the implementation and behaviour section.

A behaviour nearly identical to obstacle avoidance was created for goal seeking, the major difference being goal objects generated steering signals that moved the robot towards the object, instead of away. Since the camera signal was interpreted as an 8-bit value, where 0 corresponded to the goal being on the far left and 255 on the far right, creating a steering signal to aim for the goal was simple: the camera signal was used as is for steering.

Next, the obstacle-avoidance behaviour and goalseeking behaviour were combined. As it was considered more important to avoid collisions rather than reach goals, a weighting function was used that favoured the obstacleavoidance signal. Through experimentation, it was decided that $80 \%$ of the obstacle-avoidance-based steering signal was used, and $20 \%$ of the goal-seeking-based signal was used. This gave priority to avoiding collisions when an obstacle was observed.

\section{Goal-seeking and obstacle-avoidance results}

Only limited testing was performed for this sensor implementation. Two separate scenarios were used in which the first had the robot using only the goal-seeking behaviour, and the second involved the integrated obstacle-avoidance and goal-seeking behaviours described above.

During each set of tests, a $10-\mathrm{cm}$ tall, $13-\mathrm{cm}$ wide piece of pink foam was used as the goal colour. This colour was chosen as there were no objects of a similar colour in the test area. On start-up of the sensor pod, the camera was trained on the goal colour, after which the autonomous behaviour was initiated.

For the goal-seeking-only tests, the robot was placed in the hallway for the semi-autonomous obstacle-avoidance 
tests described in the experiment scenarios section. These tests, however, used the coloured goal with no obstacles. For six trials, the robot was placed at random angles and distances to the goal. The goal was manually held approximately $15 \mathrm{~cm}$ above the floor. Since the obstacle-avoidance behaviour was not used for these experiments (which would generate speed signals based on obstacle distance), a fixed drive speed was implemented. During each of the trials, the robot successfully actuated the steering servo motors and moved towards the goal.

Three additional sets of goal-seeking-only trials were conducted in which the goal was not visible to the robot. For the first exercises, the goal was out of the field of view and remained there; the robot drove straight, as no guidance signal was generated. A second set of experiments were conducted in which the goal started out of the field of view and then moved towards the path of the robot. For these tests, the camera noticed the goal and generated appropriate steering signals. During the third set of trials, the goal would initially be visible and then be removed from the camera's field of view. For these runs, the robot would steer towards the moving goal and then drive in a circle towards the side on which the goal was last seen. No behaviours were created to perform a seeking routine if the goal was not visible.

Later, an obstacle course was constructed similar to that described in the experiment scenarios section. This environment was used for combined goal-seeking and obstacleavoidance behaviours. Four $60-\mathrm{cm}$ tall cardboard cylinders $12 \mathrm{~cm}$ in diameter were used as obstacles.

For these tests, Whegs ${ }^{\mathrm{TM}}$ II would move along the hallway towards the goal, which was placed at the end. In most trials, it was unlikely that the goal was visible to the robot due to the visual obstruction of the obstacles. However, when the goal was not seen, the basic obstacle-avoidance behaviour caused the robot to navigate the cluttered environment. When the goal was spied, the steering signal was altered to include the new influence.

In 10 trials, the goal was found six times. The low success rate was mostly due to the obstacle-avoidance behaviour navigating past the last obstacle such that the goal was not in the field of view. Unfortunately, due to the limited memory storage capabilities of the BrainStem microcontroller, it was not possible to implement a searching behaviour. Similarly, a single obstacle collision occurred when the goal was visible, but the obstacle was to the side of the ultrasonic sensor field. Several instances of rubbing the wall also occurred due to a shallow sensing angle between the wall and the robot's path.

On one occasion, the robot drove along a path that caused it to stop moving. The chosen path did not offer a course that would allow the robot to avoid colliding with either the wall or an obstacle. As the ultrasonic sensors detected an impending collision, the speed was reduced to a point at which the robot came to a halt and didn't move further.
Table 3. Goal-seeking and obstacle-avoidance trial results.

\begin{tabular}{lc}
\hline Result & Number of times \\
\hline Goal found & $6 / 10$ \\
Obstacle collisions & $1 / 40$ \\
Minor wall collisions & $5 / 34$ \\
Robot stopped & 1 \\
\hline
\end{tabular}

\section{Conclusions}

This paper compares two distinct methods that allow small, mobile robots such as Whegs ${ }^{\mathrm{TM}}$ II to navigate obstacles, using simple exteroceptive sensors and low-end microcontrollers with limited computation capability. Rudimentary tactile antennae were used to sense a shelf as the robot approached it. Modelling cockroach behaviour, the robot decided to climb if both antennae landed on the top of the shelf and tunnel if both struck the lower surface of the shelf. If the antennae were split with one on the bottom and the other on the top of the shelf, the robot tunnelled as does the insect. Unlike the animal's antennae, the robot's antennae were relatively rigid and could become entangled in the environment. Non-contact ultrasonic sensors were explored as an alternative. When these sensors were installed in a bat-inspired configuration (a mouth-emitter and two ear-receivers), using the same microcontroller, the robot detected obstacles and circumvented those too tall to climb. When a vision-based goal-seeking system was integrated with the ultrasonic obstacle-avoidance system, the robot could seek out a desired destination while avoiding collisions. This integration of sensory-perception modalities and more complex decision-making processes - performed with a single low-end microcontroller - is the beginning of a system that is necessary for a robot to perform real-world missions in complex environments.

\section{Acknowledgement}

This material is based upon work supported by the National Science Foundation (NSF) under grant no. 0516587 and the IGERT training grant DGE 9972747, the AFOSR under grant FA955007-1-0149 and grant F08630-03-1-0003 and the NASA Langley Research Center under grant NNL06AA19G.

\section{References}

Allen TA, Quinn RD, Bachmann RJ,Ritzmann RE 2003. Abstracted biological principles applied with reduced actuation improve mobility of legged vehicles. Paper presented at: IEEE International Conference on Intelligent Robots and Systems (IROS 2003); Las Vegas, NV, USA.

Baba Y, Comer CM. 2008. Antennal motor system of the cockroach, periplaneta americana. Cell Tissue Res. 331:751762.

Bank D. 2002a. "A high-performance ultrasonic sensing system for mobile robots." In ROBOTIK 2002: Leistungsstand, Anwendungen, Visionen, Trends. VDI-Berichte Nr. 1679, VDI-Verlag, pp. 557-564. 
Bank D. 2002b. "A novel ultrasonic sensing system for autonomous mobile systems." IEEE Sensors J. 2(6):597-606.

Bell WJ. 1991. Searching behaviour: the behavioral ecology of finding resources. London: Chapman and Hall.

Blaesing B, Cruse H. 2004. Mechanisms of stick insect locomotion in a gap-crossing paradigm. J Comp Physiol A. 190:173183.

Brooks RA. 1989. "A robot that walks; emergent behavior from a carefully evolved network." Neural Comput. 1:253262.

Camhi, Johnson. 1999. "High frequency steering maneuvers mediated by tactile cues: antennal wall following in the cockroach." J Exp Biol. 202(5):631-643.

Carr CE, Konishi M. 1990. "A circuit for detection of interaural time differences in the brain stem of the barn owl." J Neurosci. 10(10):3227-3246.

Comer CM, Parks L, Halvorsen MB, Breese-Terteling A. 2003. The antennal system and cockroach evasive behavior. Part II. Stimulus identification and localization are separable antennal functions. J Comp Physiol A. 189:97-103.

Dürr V, Konig Y, Kittmann R. 2001. The antennal motor system of the stick insect Carausius morosus: anatomy and antennal movement pattern during walking. J Comp Physiol A. 187:131-144.

Dürr V, Krause A, Schmitz J, Cruse H. 2003. Neuroethological concepts and their transfer to walking machines. Int J Robot Res. 22:151-167.

Feiten W, Bauer R, Lawitsky G. 1994. "Robust obstacle avoidance in unknown and cramped environments." In Proceedings of the IEEE International Conference on Robotics and Automation (ICRA), San Diego, CA, USA. IEEE Computer Society Press, pp. 2412-2417.

Harley CM, English BA, Ritzmann RE. Forthcoming 2008. "Characterization of obstacle negotiation behaviors in the cockroach, Blaberus discoidalis." J Exp Biol.

Hartmann MJ. 2001. "Active sensing capabilities of the rat whisker system.” Auton Robots. 11:249-254.

Horiuchi T. 2005. 'Seeing' in the dark: neuromorphic VLSI modeling of bat echolocation. IEEE Sig Process Mag. 22(5):134139.

Horiuchi T, Hynna KM. 2001. "Spike-based modeling of the ILD system in the echolocating bat". Neural Networ. 14:755762.

Horchler AD, Reeve RE, Webb BH, Quinn RD.2003. "Robot phonotaxis in the wild: a biologically inspired approach to outdoor sound localization". Paper presented at: 11th International Conference on Advanced Robotics (ICAR'03); Coimbra, Portugal.

Jeanson R, Deneubourg JL. 2007. Conspecific attraction and shelter selection in gregarious insects. Am Nat. 170:47-58.

Jindrich DL, Full RJ. 1999. Many-legged maneuverability: dynamics of turning in hexapods. J Exp Biol. 202:16031623.

Jones G. 2005. "Echolocation”. Curr Biol. 15(13):R484-R488.

Krause AF, Dürr, V. 2004. Tactile efficiency of insect antennae with two hinge joints. Biol Cybern. 91:168-181.

Lawitsky G, Feiten W, Möller M. 1995. "Sonar sensing for lowcost indoor mobility." Robot Automat Syst. 14:149-157.

Lee J, Sponberg S, Loh O, Cowan N. 2008. "Templates and anchors for antenna-based wall following in cockroaches and robots." IEEE Trans Robot. 24(6):130-143.
Lewinger WA, Harley CM, Ritzmann RE., Branicky MS, Quinn RD. 2005a."Insect-like antennal sensing for climbing and tunneling behavior in a biologically-inspired mobile robot." Proceedings of the IEEE International Conference on Robotics and Automation (ICRA '05), Barcelona, Spain, April 18-22, 2005.

Lewinger WA, Harley CM et al. 2005b. "Insect-like antennal sensing for climbing and tunneling behavior in a biologicallyinspired mobile robot." Video Proceedings of the IEEE International Conference on Robotics and Automation (ICRA '05); 2005 April 18-22; Barcelona, Spain.

Loeb GE, Brown IE, Cheng EJ. 1999. A hierarchical foundation for models of sensorimotor control. Exp Brain Res. 126:1-18.

Lund HH, Webb B, Hallam J. 1998. "Physical and temporal scaling considerations in a robot model of cricket calling song preference." Artif Life. 4:95-107, 1998.

Martin KD. 1995. "Estimating azimuth and elevation from interaural differences." Paper presented at the IEEE Mohonk Workshop on Applications of Signal Processing to Acoustics and Audio, Mohonk, NY, US.

Martin-Alvarez A, De Peuter W, Hillebrand J, Putz P, Matthyssen A, De Weerd JF. 1996. Walking robots for planetary exploration missions. Paper presented at the Second World Automation Congress (WAC '96); Montpellier, France.

$\mathrm{Mu}$ L, Ritzmann RE. 2005. "Kinematics and motor activity during tethered walking and turning in the cockroach, Blaberus discoidalis." J Comp Physiol A. 191:1037-1054.

Okada J, Toh Y. 1998. Shade response in the escape behavior of the cockroach Periplaneta Americana. Zool Sci. 15:831-835.

Okada J, Toh Y. 2000. "The role of antennal hair plates in objectguided tactile orientation of the cockroach (Periplaneta americana)." J Comp Physiol A. 186(9):849-857.

Okada J, Toh Y. 2001. "Peripheral representation of antennal orientation by the scapal hair plate of the cockroach Periplaneta americana." J Exp Biol. 204:4301-4309.

Pelletier Y, McLoed C. 1994. Obstacle perception by insect antennae during terrestrial locomotion. Physiol Entomol. 19:360 362.

Quinn RD, Nelson GM, Ritzmann RE, et al. 2003. "Parallel strategies for implementing biological principles into mobile robots." Int J Robot Res. 22:169-186.

Saranli U, Buehler M, Koditschek D. 2001. RHex a simple and highly mobile hexapod robot. Int J Robot Res. 20(7): 616631.

Shi R, Horiuchi T. 2004. "A VLSI model of the bat lateral superior olive for azimuthal echolocation." Proceedings of the 2004 International Symposium on Circuits and Systems (ISCAS '04).

Staudacher EM, Gebhardt M, Dürr V. 2005. Antennal movements and mechanoreception: neurobiology of active tactile sensors. Adv Insect Physiol. 32:50-172.

Watson JT, Ritzmann RE. 1998. "Leg kinematics and muscle activity during treadmill running in the cockroach", blaberus discoidalis: I. slow running." J Comp physiol. 182:11-22.

Watson JT, Ritzmann RE, Zill SN, et al. 2002. Control of obstacle climbing in the cockroach, Blaberus discoidalis. Part I. Kinematics. J Comp Physiol A. 188:39-53.

Ye S, Leung V, Khan A, et al. 2003. The antennal system and cockroach evasive behavior. Part I. Roles for visual and mechanosensory cues in the response. J Comp Physiol A. 189: 89-96. 

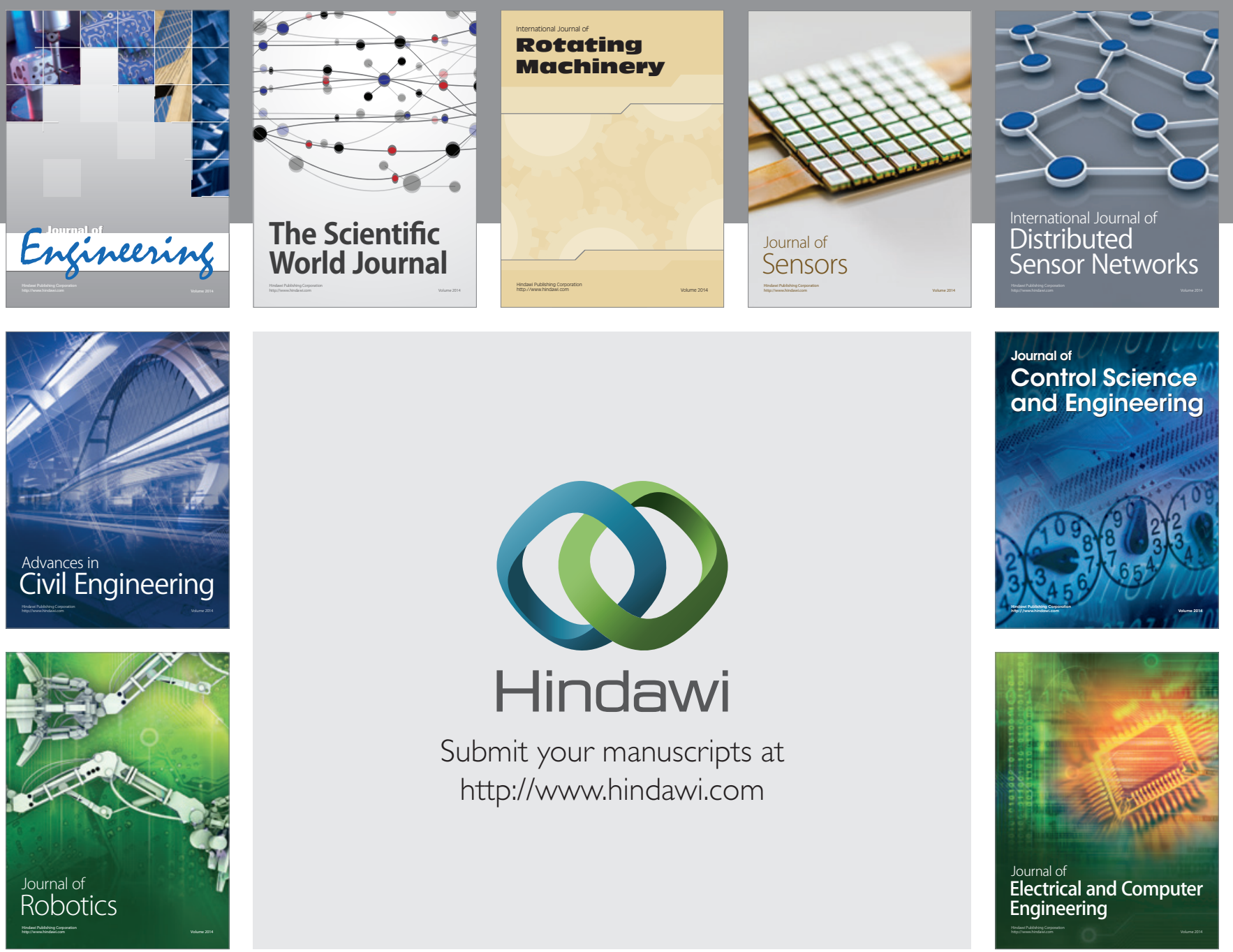

Submit your manuscripts at

http://www.hindawi.com
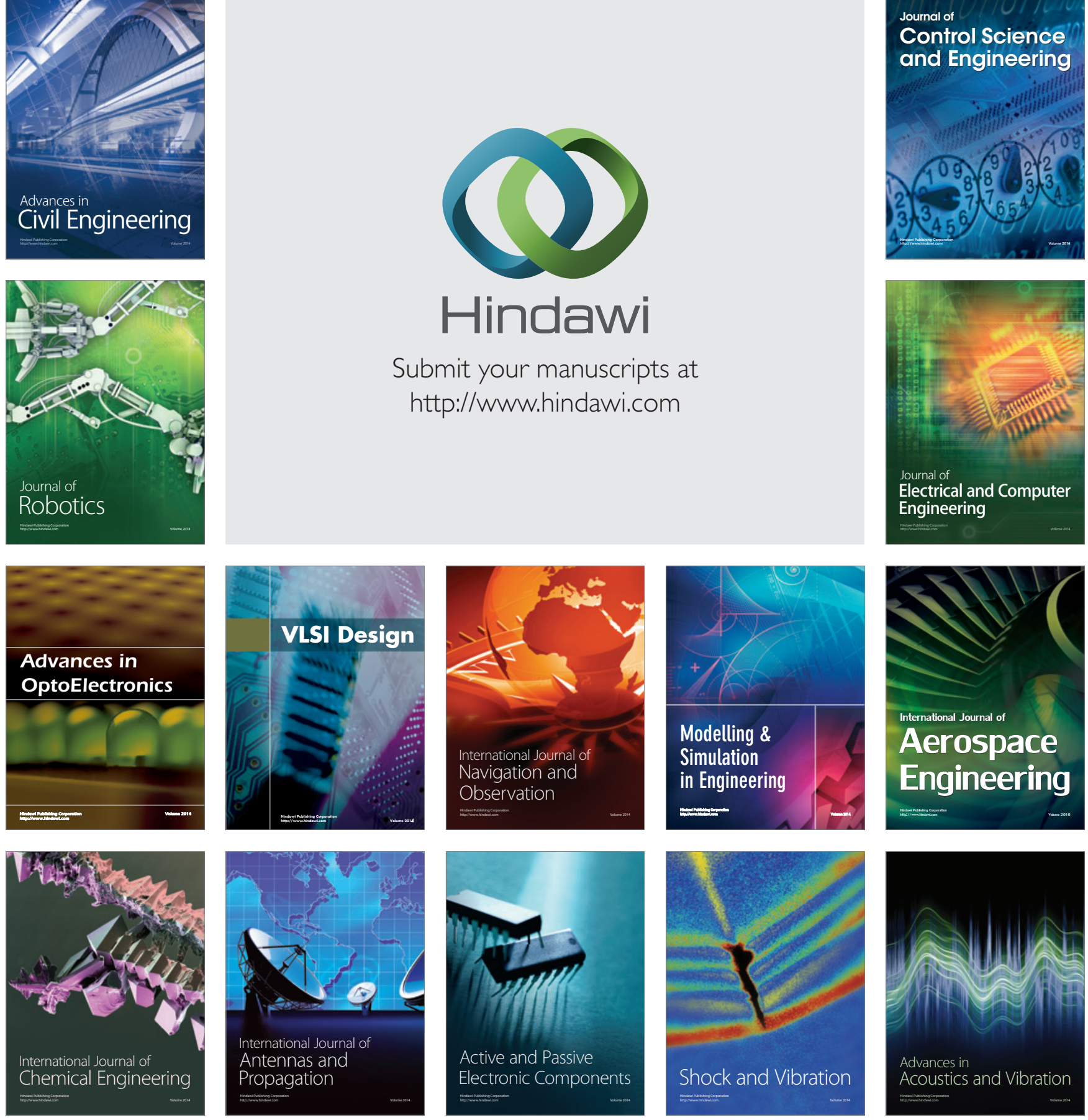